\title{
Die zeitliche Entwicklung von Abschlussprüferhonoraren in Deutschland - Eine empirische Untersuchung der Jahre 2009 bis 2013
}
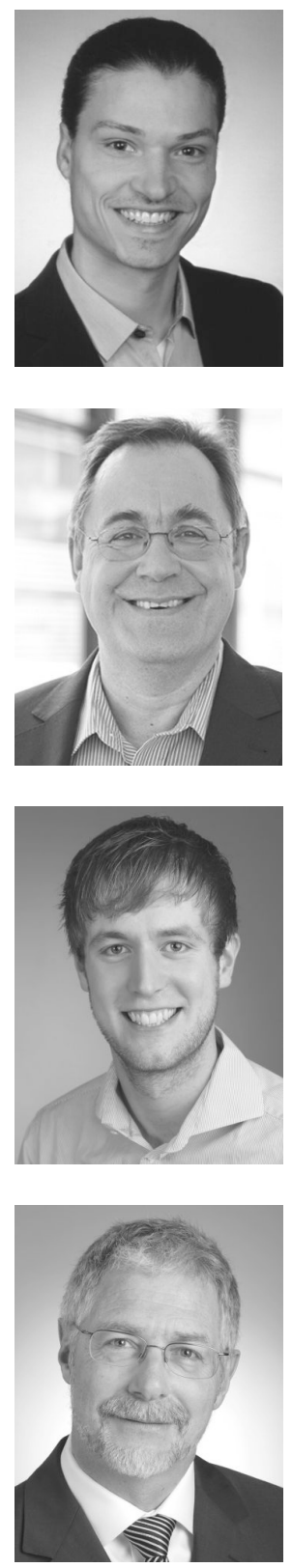

Heiko Laudemann, Reiner Quick, Florian Schmidt und Bent Warming-Rasmussen

Auswahlindizes; Beratungshonorare; Prüfungshonorare; Prüfungsmarkt; Unabhängigkeit des Abschlussprüfers; Wirtschaftsprüfung Audit fees; Auditing; Audit market; Auditor independence; German stock market; Non-audit fees

Seit 2005 besteht die Pflicht, die Honorare des Abschlussprüfers nach Honorarkategorien differenziert im Anhang zu publizieren. Die dadurch ermöglichten Einblicke erlauben es Stakeholdern, die Unabhängigkeit des Abschlussprüfers zu beurteilen. Das Ziel des vorliegenden Beitrags ist es, die Abschlussprüferhonorare für die wichtigsten deutschen Börsenindizes in ihrer zeitlichen Entwicklung und ihrer Zusammensetzung für den Zeitraum von 2009 bis 2013 $\mathrm{zu}$ analysieren. Den Honoraren für Abschlussprüfungsleistungen kommt mit Abstand die grösste Bedeutung zu, obwohl sie im Zeitablauf sinken. Diese Entwicklung ist allerdings ausschliesslich durch DAX30-Unternehmen getrieben. Der Anteil der Honorare für Nichtprüfungsleistungen ist im DAX30 aber auch im SDAX im Hinblick auf die Unabhängigkeit der Abschlussprüfer bedenklich.

Since 2005, German audit clients are obliged to publish auditor fees in the notes to their financial statements. The fees have to be differentiated into four categories: audit services, andit-related services, tax advisory services, and other services. Such a disclosure enables stakeholders to evaluate auditor independence. This paper analyses the temporal development of auditor fees as well as their composition for the period 2009-2013. Fees for audit services are by far the highest and decline over time. However, this development is exclusively driven by DAX30 companies. With regard to auditor independence, the share of non-audit fees is critical for DAX30 but also for SDAX companies.

\section{Einleitung}

Ausgelöst durch Unternehmensschieflagen und Bilanzskandale und dem damit verbundenen Vorwurf, dass auch die Abschlussprüfer 
versagt hätten, wurden in den vergangenen Jahren zahlreiche Reformen im Bereich der Prüfungsnormen auf den Weg gebracht, welche die Prüfungsqualität im Allgemeinen und die Unabhängigkeit des Abschlussprüfers im Besonderen stärken sollten. Ein bedeutendes Element dieser neuen Regelungen stellt die Pflicht zum Ausweis des für den Abschlussprüfer angefallenen Honorars im Anhang bzw. Konzernanhang dar (Petersen/Zwirner 2008b, 280). So schreiben die $\mathbb{S} \$ 285$ Nr. 17 und 314 Abs. 1 Nr. 9 HGB eine Aufteilung der von dem gesetzlichen Abschlussprüfer erbrachten Leistungen in die vier Kategorien Honorare für Abschlussprüfungsleistungen, andere Bestätigungsleistungen, Steuerberatungsleitungen und sonstige Leistungen vor. Der Abschlussprüfer hat im Vergleich zu den Adressaten seines Prüfungsergebnisses einen Informationsvorsprung und könnte daher versucht sein, seine Unabhängigkeit gegenüber dem Management aufzugeben und Zahlungen dafür zu akzeptieren, dass er in seinem Prüfungsbericht aufgedeckte Unregelmässigkeiten verschweigt. Überhöhte Prüfungshonorare oder Beratungshonorare verleihen solchen Zahlungen einen legalen Charakter (Antle 1984, 16). Diese Offenlegungspflichten erlauben nunmehr den Abschlussadressaten eine eigene Einschätzung und Interpretation der Honorare im Sinne möglicher Implikationen für die Prüfungsqualität und die Unabhängigkeit des Abschlussprüfers.

Grundsätzlich ist das Prüfungshonorar frei vereinbar. Es gibt keine Honorarordnung für Wirtschaftsprüfer (Velte 2009, 1229; Ebke 2013, Rn. 31; Schmidt/Heinz 2014, Rn. 16). Handels- und aktienrechtlich werden keine Konkretisierungen hinsichtlich des Honorars des Abschlussprüfers aufgeführt, wobei $\mathbb{S} 55$ Abs. 1 S. 1 WPO ein Erfolgshonorar ausschliesst und die Berufssatzung der Wirtschaftsprüfer zur Sicherstellung der Qualität der beruflichen Tätigkeit zumindest eine angemessene Vergütung fordert ( $\$ 27$ BS WP/vBP). Ein Pauschalhonorar darf nur vereinbart werden, wenn festgelegt ist, dass das Honorar bei Eintritt unvorhergesehener Umstände zu erhöhen ist ( $\$ 27$ Abs. 2 BS WP/vBP). Wie eine Honorarumfrage der Wirtschaftsprüferkammer ergab, sind Pauschalhonorare weit verbreitet und von der Escape-Klausel wird nur selten Gebrauch gemacht (WPK 2011). Damit erhöht sich die Gefahr, dass das Honorar zu niedrig angesetzt wird, Prüfungshandlungen nicht im ausreichenden Mass durchgeführt werden und so keine hinreichende Prüfungssicherheit erlangt wird (Velte 2009, 1230-1231; Umlauf 2013, 116).

Erwähnenswert ist in diesem Zusammenhang auch die Verordnung des Europäischen Parlaments und des Rates vom 16. April 2014 (EU-Parlament \&o Rat der EU 2014) über spezifische Anforderungen an die Abschlussprüfung bei Unternehmen von öffentlichem Interesse. Diese enthält nicht nur eine umfassende Liste mit Nichtprüfungsleistungen, die der Abschlussprüfer künftig nicht mehr für das geprüfte Unternehmen erbringen darf (Artikel 5), sondern begrenzt auch die Honorare für Nichtprüfungsleistungen. Wenn ein $\mathrm{Ab}$ schlussprüfer oder eine Prüfungsgesellschaft für einen Zeitraum von drei oder mehr aufeinanderfolgenden Geschäftsjahren für ein geprüftes Unternehmen noch erlaubte Nichtprüfungsleistungen erbringt, werden die Gesamthonorare für diese Leistungen auf maximal $70 \%$ des Durchschnitts der in den letzten drei aufeinanderfolgenden Geschäftsjahren für die Abschlussprüfungen gezahlten Honorare begrenzt (Artikel 4 Abs. 2). Die neue Verordnung hat unmittelbare Wirkung und gilt ab dem 17. Juni 2016. Schliesslich ist darauf hinzuweisen, dass nach $\mathbb{3} 319 \mathrm{a}$ Abs. $1 \mathrm{Nr}$. 1 HGB ein Wirtschaftsprüfer bzw. eine Wirtschaftsprüfungsgesellschaft (WPG) von der Abschlussprüfung eines kapitalmarktorientierten Unternehmens ausgeschlossen ist, wenn er bzw. sie in den letzten fünf Jahren jeweils mehr als $15 \%$ der Gesamteinnahmen aus seiner bzw. ihrer beruflichen Tätigkeit von der 
zu prüfenden Gesellschaft bezogen hat und dies auch im laufenden Geschäftsjahr zu erwarten ist.

Analysen zu den Honoraren von Abschlussprüfern sind ein beliebter Gegenstand wissenschaftlicher Studien. Diese behandeln u.a. die Fragen, ob das an den Abschlussprüfer gezahlte Honorar in einer angemessenen Relation zu der erbrachten Leistung steht, welche Faktoren die Höhe der Prüfungshonorare beeinflussen, inwiefern die Prüfungshonorare Rückschlüsse auf die Prüfungsqualität zulassen oder nach der Verhältnismässigkeit von Prüfungs- und Beratungsleistungen (vgl. hierzu bspw. Niehus 2002; Lenz/Bauer 2004; Polt/Winter 2004; Lenz et al. 2006; Zimmermann 2006). So kann eine parallele Prüfung und Beratung die Unabhängigkeit des Abschlussprüfers gefährden, etwa weil durch die Beratung eine persönliche Bindung zwischen Prüfer und Funktionsträgern des Mandanten entsteht, weil der Abschlussprüfer mit den Ergebnissen seiner eigenen Beratungstätigkeit konfrontiert wird oder weil durch die zusätzlichen Beratungshonorare die Gefahr einer finanziellen Abhängigkeit steigt (Quick 2006, 43). Weitere Studien gehen der Frage nach dem Verhältnis zwischen Beratungs- und Prüfungshonoraren nach bzw. analysieren die Entwicklung der Honorare im Zeitablauf (vgl. hierzu Kapitel 3).

Ziel dieses Beitrags ist es, die Art und Höhe der an Abschlussprüfer von börsennotierten Unternehmen gezahlten Honorare in Deutschland empirisch zu untersuchen. Dadurch lassen sich Rückschlüsse ziehen auf die Frage, ob eine hinreichende Prüfungsqualität, wie sie durch die Prüfungsnormen (ISA 200; ISA 300; IDW PS 200) gefordert wird, erlangt wird. Wenngleich Prüfungshonorare nicht direkt die Prüfungsqualität messen, stellen sie ein Qualitätssurrogat dar, das in der Forschung breiten Einsatz findet. ${ }^{1}$ Die hohe Konzentration auf den Prüfungsmärkten gibt Anlass zur Besorgnis, dass diese den Wettbewerb einschränkt und Prüfungsgesellschaften überhöhte Prüfungshonorare durchsetzen. Insofern lassen die beobachteten Prüfungshonorare mögliche Auswirkungen dieser Wettbewerbssituation erkennen. Hohe Beratungshonorare gefährden die Unabhängigkeit des Abschlussprüfers bzw. können eine Besorgnis der Befangenheit auslösen. Daher kann sowohl aus der absoluten als auch der relativen Höhe der Beratungshonorare auf das Ausmass einer möglichen Unabhängigkeitsbeeinträchtigung geschlossen werden. Da verschiedene Beratungsleistungen unterschiedliche Unabhängigkeitswahrnehmungen auslösen, bietet auch die $\mathrm{Zu}$ sammensetzung der Nichtprüfungshonorare einen Einblick in die Unabhängigkeitssituation der Abschlussprüfer (Quick/Warming-Rasmussen 2009). Dabei soll der Fokus auf der Entwicklung im Zeitverlauf der Jahre 2009 bis 2013 liegen. Hierfür werden die Unternehmen der Auswahlindizes DAX30, MDAX, TecDAX und SDAX des Prime Standards der Frankfurter Börse betrachtet, wobei auch die einzelnen DAX-Indizes eine differenzierte Einzelbetrachtung erfahren. Obwohl die Konzentration im Börsensegment DAX30 am höchsten ist, zeigen die Ergebnisse, dass die Prüfungshonorare dort im Zeitablauf zurückgegangen sind. Die insbesondere von Normengebern häufig geäusserte Befürchtung, dass eine hohe Anbieterkonzentration zu Lasten des Wettbewerbs gehe, lässt sich aus den vorliegenden Daten nicht belegen. Während im MDAX für die Honorare für Abschlussprüfungsleistungen kein eindeutiger Trend identifiziert werden kann, verlaufen diese im SDAX und im TecDAX ansteigend. In Relation zu den Prüfungshonoraren sind die Honorare für Nichtprüfungsleistungen im DAX30 mit einem durchschnittlichen Anteil von $75 \%$ besonders hoch. Die von der EU als kritisch angesehene Grenze von $70 \%$ wird somit häufig

1 Zur einer ausführlichen Diskussion vgl. DeFond/Zhang (2014). 
überschritten und es besteht die Gefahr einer finanziellen Abhängigkeit. Aufgrund der $\mathrm{Pu}$ blizität der Abschlussprüferhonorare besteht zudem die Gefahr, dass Adressaten des geprüften Jahresabschlusses den Abschlussprüfer als abhängig wahrnehmen, was zu Lasten der Glaubwürdigkeit der Finanzberichterstattung ginge. Auch im SDAX ist der Anteil der Beratungshonorare mit knapp $68 \%$ noch recht hoch, sodass ähnliche Unabhängigkeitsprobleme zu vermuten sind, wohingegen der MDAX und der TecDAX durch moderatere Werte gekennzeichnet sind.

Dieser Beitrag trägt aus mehrfachen Gründen zum Erkenntnisfortschritt bei. Er beschränkt sich nicht nur auf eine Untersuchungsperiode, sondern analysiert ein Fünfjahreszeitraum und ist damit geeignet, Entwicklungstendenzen im Zeitablauf zu identifizieren. Darüber hinaus handelt es sich um die erste Studie, die sich mit der Zusammensetzung und Entwicklung von Abschlussprüferhonoraren nach Änderung der diesbezüglichen $\mathrm{Pu}$ blizitätspflichten im Jahr 2009 auseinandersetzt. Ausserdem geht er der Frage nach, auf welche Ursachen Ausreisser bzw. Brüche in der Entwicklung zurückzuführen sind. Aus aktuellem Anlass beschäftigt er sich zudem mit der Frage, inwieweit die von der EU formulierte kritische Honorargrenze für Nichtprüfungsleistungen überschritten wird.

Der Beitrag ist wie folgt aufgebaut. In Kapitel 2 erfolgt eine Diskussion der Honorarpublizität nach HGB. Darauf folgend informiert Kapitel 3 über die bisherigen Studien zur Zusammensetzung und zeitlichen Entwicklung der Abschlussprüferhonorare für den deutschen Prüfungsmarkt. In Kapitel 4 schliesst sich ein Überblick über die Stichprobe der empirischen Erhebung an, d.h. es stellt den betrachteten Teilmarkt dar und erklärt, aus welchen Gründen Bereinigungen der Stichprobe vorgenommen werden mussten. Daran anschliessend werden in Kapitel 5 die Ergebnisse der Honoraruntersuchung vorgestellt. Diese beziehen sich nicht nur auf die gesamte Stichprobe. Vielmehr erfolgt auch eine nach Auswahlindizes differenzierte Analyse. Kapitel 6 beschliesst den Beitrag mit einer kurzen $\mathrm{Zu}$ sammenfassung sowie einem Ausblick auf künftige Forschungsaktivitäten.

\section{Publizität der Abschlussprüferhonorare}

\subsection{Entwicklung der Normen}

Mit der Überarbeitung der 8. EU-Richtlinie sind (mit Art. 49) Änderungen der 4. EURichtlinie und der 7. EU-Richtlinie einhergegangen. Letztere wurden u.a. um den Zusatz erweitert, dass im Anhang Gesamthonorare für Leistungen des gesetzlichen Abschlussprüfers anzugeben sind (EU-Parlament \& Rat der EU 2006, Art. 49).

Durch das Bilanzrechtsreformgesetz (BilReG) vom 4.12.2004 wurde auch in Deutschland die Pflicht, Prüferhonorare offen zu legen, im HGB verankert (Lenz et al. 2006, 1787; Sattler 2011, 6). Ziel der Offenlegung ist eine verbesserte Einschätzung der Unabhängigkeit und Objektivität des Abschlussprüfers durch die Adressaten des Jahresabschlusses (Wollmert et al. 2010, 123). Mit $\$ 285$ Satz 1 Nr. 17 HGB a.F. forderte der Gesetzgeber die Offenlegung der von Abschlussprüfern im Sinne des $\mathbb{} 319$ Abs. 1 Satz 1 und 2 HGB im Geschäftsjahr angefallenen Honorare im Anhang eines Jahresabschlusses. Diese waren in die Kategorien „Abschlussprüfung “, „sonstige Bestätigungs- oder Bewertungsleistungen“, „Steuerberatungsleistungen“ und „sonstige Leistungen“ aufzuschlüsseln. Analog dazu hatte nach $\$ 314$ Abs. 1 Nr. 9 HGB a.F. die Offenlegung der im Geschäftsjahr angefallenen Honorare für Konzernabschlussprüfer im Sinne des $\$ 319$ Abs. 1 Satz 1 und 2 HGB im Konzernanhang der Muttergesellschaften zu erfolgen. Die mit der Einführung des 
BilReG einhergehenden Neuerungen griffen dem europäischen Gesetzgeber vor (Petersen/ Zwirner 2008a, 51; Petersen/Zwirner 2008c, 967). Der Gesetzgeber unterliess eine weitere Erläuterung der Zuordnung der Leistungen zu den einzelnen Honorarkategorien, sodass dies ergänzend durch den Berufsstand mit dem Rechnungslegungshinweis IDW RH HFA 1.006 vorgenommen wurde. Beide Publizitätsvorschriften galten, entgegen dem Referenten- und Gesetzesentwurf, nur für Unternehmen, die einen organisierten Markt i.S.d. $\mathbb{} 2$ Abs. 5 WpHG in Anspruch nehmen (Krauß 2008, 45-46). Gemäss Art. 58 Abs. 3 Satz 1 EGHGB fanden diese Vorschriften erstmals auf nach dem 31.12.2004 beginnende Geschäftsjahre bzw. Rumpf-Geschäftsjahre Anwendung. Somit waren die Angaben der Honorare erstmalig in Jahres- und Konzernabschlüssen zum 31.12.2005 zu finden (Bischof 2006, 706).

Mit der Einführung des Bilanzrechtsmodernisierungsgesetzes (BilMoG) am 25.5.2009 (das BilMoG trat am 29.5.2009 in Kraft) nahm der Gesetzgeber einige Änderungen der bereits bestehenden Publizitätspflichten vor, die erstmals auf Jahres- und Konzernabschlüsse für Geschäftsjahre, die nach dem 31. Dezember 2008 beginnen, anzuwenden sind (Artikel 66 Abs. 2 Satz 1 des EGHGB). Die Pflicht zur Honoraroffenlegung ist weiterhin in zwei handelsrechtlichen Vorschriften normiert. So sind die Honorarangaben nach $\mathbb{} 285$ Nr. 17 HGB im Anhang des Jahresabschlusses und nach $\mathbb{3} 314$ Abs. 1 Nr. 9 HGB im Anhang des Konzernabschlusses aufzulisten. Für den Anhang des Jahresabschlusses von Kapitalgesellschaften fordert $\mathbb{2} 285 \mathrm{Nr}$. 17 HGB eine Angabe des für das Geschäftsjahr berechneten Gesamthonorars des Abschlussprüfers, aufgeschlüsselt in die vier Kategorien „Abschlussprüfungsleistungen“, „andere Bestätigungsleistungen“, „Steuerberatungsleistungen“ und „sonstige Leistungen“. Während also in der Vergangenheit eine Veröffentlichung der im Geschäftsjahr angefallenen Honorare gefordert war, zielt der Gesetzgeber nunmehr auf die für das Geschäftsjahr berechneten Honorare ab, d.h., die Periodenzuordnung der Honorare hat sich geändert. Eine Angabe der Honorare ist nicht erforderlich, sofern diese bereits in einem das Unternehmen einbeziehenden Konzernabschluss enthalten sind. Analog dazu ist das für das Geschäftsjahr berechnete Konzernabschlussprüferhonorar im Konzernanhang durch $\mathbb{S} 314$ Abs. 1 Nr. 9 HGB in den selben vier Leistungskategorien anzugeben. Der Gesetzgeber unterlässt weiterhin eine genaue Abgrenzung der Leistungsarten.

Grundsätzlich sind nunmehr alle publizitätspflichtigen Unternehmen zu einer Angabe im Anhang verpflichtet, d.h. im Vergleich zur Regelung des BilReG, welches eine Begrenzung auf Unternehmen, die einen organisierten Markt nach $\$ 2$ Abs. 5 WpHG in Anspruch nehmen, vorsah, ist die Neuregelung weiter gefasst. Die Pflicht zur Angabe der Honorare im Anhang eines Konzern- oder Einzelabschlusses schliesst somit Kapitalgesellschaften und haftungsbeschränkte Personenhandelsgesellschaften i.S.d. $\$ 264$ a Abs. 1 HGB ein (IDW RS HFA 36 2010; Rn. 1). Kleine Kapitalgesellschaften i.S.d. $\mathbb{} 267$ Abs. 1 HGB, die nach

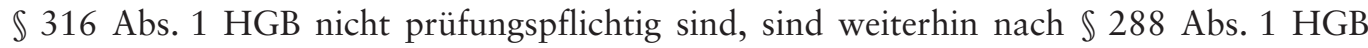
von der Angabepflicht befreit. Hinzugekommen ist die Befreiung für mittelgrosse Kapitalgesellschaften, welche jedoch auf Aufforderung der WPK die Honorardaten an diese zu übermitteln haben ( $\mathbb{2} 288$ Abs. 2 Satz 3 HGB) (IDW RS HFA 36 2010, Rn. 3; Kessler 2013, Rn. 193; Grottel 2014a, Rn. 290). Des Weiteren sind auch Unternehmen, die dem Publizitätsgesetz unterliegen und zur Aufstellung eines Anhangs verpflichtet sind, zur Angabe verpflichtet. Dies gilt auch für Kredit- und Finanzdienstleistungsinstitute sowie für Versicherungsunternehmen, unabhängig von ihrer Rechtsform (Kessler 2013, Rn. 193; 
Umlauf 2013, 101). Ferner muss auch bei einem freiwillig nach IFRS aufgestellten Einzelabschluss, der anstelle des handelsrechtlichen Jahresabschlusses publiziert wird, das Honorar im Anhang angegeben werden ( $\$ 325$ Abs. 2a HGB) (Umlauf 2013, 102). Unterlässt das angabepflichtige Unternehmen die Auflistung der Gesamthonorare im Anhang, so ist der Bestätigungsvermerk nur eingeschränkt zu erteilen (Kessler 2013, Rn. 202).

\subsection{Honorarkategorien}

Der Kategorie „Abschlussprüfungsleistungen“ sind grundsätzlich nur die Leistungen zuzuordnen, welche der Abschlussprüfer für die Prüfung des Jahresabschlusses erbringt (IDW RS HFA 36 2010, Rn. 12; Grottel 2014a, Rn. 301). Somit werden u.a. die vom Abschlussprüfer zwingend durchzuführenden Prüfungen des Jahresabschlusses und des Lageberichts (i.S.d. $\$ 316$ Abs. 1 u 2 HGB) eingeschlossen. Die Kategorie beinhaltet auch Leistungen für eine erforderliche Nachtragsprüfung ( $\$ 316$ Abs. 3 HGB) sowie die Prüfung des IFRS-Einzelabschlusses ( $\$ 324$ a Abs. 1 HGB i.V.m. $\$ 316$ HBG) und des Abhängigkeitsberichts

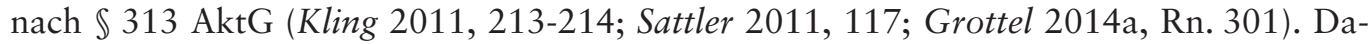
bei werden dieser Kategorie neben den Leistungen für die gesetzliche Abschlussprüfung gemäss $\$ 316$ Abs. $1 \mathrm{HGB}$ auch die mit der Abschlussprüfung in sachlichem Zusammenhang stehenden Leistungen zugeordnet (Kessler 2013, Rn. 198; Umlauf 2013, 110-111).

Die Kategorie „andere Bestätigungsleistungen“ umfasst alle übrigen Leistungen i.S.d. \2 Abs. 1 WPO, die zwar vom Abschlussprüfer durchgeführt wurden, aber lediglich im zeitlichen Zusammenhang mit oder in Ergänzung zur Abschlussprüfung stehen. Diese können freiwillig beauftragte Prüfungen analog zu $\$ 53 \mathrm{HGrG}$ oder die freiwillige Prüfung des Risikofrüherkennungssystems nach $\$ 317$ Abs. 4 HGB darstellen (Bischof 2006, 711; IDW RS HFA 36 2010, Rn. 13; Kling 2011, 214; Umlauf 2013, 111). Dabei handelt es sich um Leistungen, bei denen der bestellte Abschlussprüfer gemäss $\mathbb{} 48$ Abs. 1 WPO i.V.m. $\mathbb{} 2$ Abs. 1 WPO das Berufssiegel führen kann bzw. muss. Im Gegensatz zu den Abschlussprüfungsleistungen sind diese nicht zwingend durch den gesetzlich bestellten Abschlussprüfer vorzunehmen, sondern können auch von anderen WP-Praxen durchgeführt werden (Bischof 2006, 711; Umlauf 2013, 112). Auszuweisen sind jedoch nur die Leistungen, die von dem gesetzlich bestellten Abschlussprüfer getätigt wurden. Im Vergleich zur Rechtslage vor der Einführung des BilMoG sind Bewertungsleistungen nunmehr nicht mehr dieser Kategorie, sondern vielmehr den „sonstigen Leistungen“ zugeordnet (Wulf 2010, 408-409; Kling 2011, 2014).

Unter die „Steuerberatungsleistungen“ fallen sowohl die Honorare für erbrachte Steuerdeklarations- als auch für Steuergestaltungsberatungsleistungen (Petersen/Zwirner 2008b, 283; IDW RS HFA 36 2010, Rn. 14; Kling 2011, 214). Ferner zählen hierzu Auskünfte und Gutachten hinsichtlich steuerlicher Sachverhalte sowie Vertretungen in steuerlichen Angelegenheiten vor Finanzgerichten; d.h. es werden fast alle Leistungen einbezogen, die im Anwendungsbereich des $\mathbb{} 1$ StBerG liegen (Bischof 2006, 711; Kling 2011, 214; Umlauf 2013, 112).

Die „sonstigen Leistungen“ stellen einen Sammelposten für alle Leistungen dar, die sich nicht in die anderen Kategorien zuordnen lassen. Dabei müssen die Leistungen mit der Durchführung der Abschlussprüfung i.S. der $\mathbb{S} \mathbb{3 1 9}$, 319a HGB vereinbar sein. Hierzu gehören z.B. Honoraraufwendungen für Schulungsmassnahmen, prüfungsnahe Beratung, Beratung im IT-System-Bereich, Vorschläge zur Beseitigung von Fehlern oder Lücken im Jah- 
resabschluss, Leistungen im Rahmen einer „financial due diligence“ oder Beratungsleistungen bei der Umstellung der Rechnungslegung auf IFRS.

\subsection{Abgrenzungsfragen}

Der Begriff des Abschlussprüfers schliesst keine dem Abschlussprüfer nahestehenden Personen oder mit ihm verbundene Unternehmen ein. Somit sind keine Angaben zu dem internationalen Verbund oder Netzwerk, in welchem der Abschlussprüfer agiert, erforderlich (IDW RS HFA 36 2010, Rn. 7; Kling 2011, 212). Bischof (2006) empfiehlt jedoch vor dem Hintergrund der Unabhängigkeit des Abschlussprüfers und der damit verbundenen Befangenheitsvermutung eine Ausweitung der Angabepflicht auf Honorare anderer Rechtsträger, sofern diese im Rahmen der Prüfung von Ausschlussgründen nach $\mathbb{S} \mathbb{S} 319$, 319a, 319b HGB zu berücksichtigen sind. Eine vergleichbare Regelung fordert auch der Deutsche Corporate Governance Kodex, indem er eine Unabhängigkeitserklärung des Abschlussprüfers gegenüber dem Aufsichtsrat des zu prüfenden Unternehmens verlangt, in welcher der erweiterte Unternehmenskreis des Abschlussprüfers offenzulegen ist. Auch Kling (2011) spricht sich für eine Kennzeichnung der Honorare für Leistungen aus, die von mit dem Abschlussprüfer verbundenen Unternehmen i.S.v. $\$ 271$ Abs. 2 HGB erbracht worden sind. Der Vorschlag zielt dabei auf einen Davon-Eintrag ab (Kling 2011, 212). ${ }^{2}$ Demgegenüber argumentiert Grottel (2014a) mit der Klarheit der handelsrechtlichen Norm, welche nur die Honorare des gesetzlichen Abschlussprüfers vorsieht. Dieser Auffassung ist auch der Gesetzgeber, die er in seiner Begründung zum Regierungsentwurf des BilMoG klar stellt (BT-Drucksache 16/10067 2008, 70).

Nach $\mathbb{\$} 285$ Nr. 17 und 314 Abs. 1 Nr. 9 HGB ist das vom Abschlussprüfer für das Geschäftsjahr berechnete Gesamthonorar anzugeben. Als Gesamthonorar ist dabei die Gesamtvergütung für alle Leistungen des Abschlussprüfers für die berichtende Gesellschaft zu verstehen (Grottel 2014a, Rn. 291). Es schliesst auch den berechneten Auslagenersatz wie z.B. Tage- und Übernachtungsgelder, Fahrt- und Nebenkosten sowie Berichts- und Schreibkosten ein, jedoch nicht die entrichtete Umsatzsteuer (BT-Drucksache 16/10067 2008, 70; IDW RS HFS 36 2010, Rn. 10; Grottel 2014a, Rn. 291). Petersen/Zwirner (2008b) empfehlen zur Verdeutlichung der Entwicklung der Honorare im Zeitablauf eine Angabe der Vorjahreswerte und für eine bestmögliche Transparenz der Angaben eine verbale Zuordnung der Beträge zu den einzelnen Kategorien (Petersen/Zwirner 2008b, 281). ${ }^{3}$ Während die alte Fassung des $\mathbb{2} 285$ Satz 1 Nr. 17 HGB noch das im Geschäftsjahr als Aufwand erfasste Honorar forderte, gibt die aktuelle Norm den Terminus für das im Geschäftsjahr berechnete Gesamthonorar an. IDW RS HFA 36 stellt klar, dass es sich bei dem Gesamthonorar nicht um das bis zum Abschlussstichtag in Rechnung gestellte Honorar, sondern um das auf das Geschäftsjahr entfallene Honorar handelt. Somit ist der Zeitpunkt der Honorarvereinbarung und der noch offenen bzw. bereits getätigten Honorarzahlungen irrelevant. Es muss lediglich die Zuordnung auf das zu prüfende Geschäftsjahr berücksichtigt werden (Wulff 2010, 408; Grottel 2014a, Rn. 292). ${ }^{4}$ Zumeist fällt der Hauptteil der gesetzlichen Abschlussprüferleistungen erst nach dem abgelaufenen Geschäftsjahr an, wobei

2 Die Ausfassung zu einer erweiterten Auslegung der Norm und einer freiwilligen Angabe der Honorare teilen u.a. Lenz et al. (2006), 1788; Petersen/Zwirner (2008b), 280; Pfitzer et al. (2008), 97; IDW RS HFA 36 (2010), Rn. 7.

3 Vgl. zur Empfehlung der verbalen Beschreibung auch Bischof (2006), 710.

4 Siehe auch hierzu den Gestaltungsspielraum vor BilMoG; Zimmermann (2006). 
die Schlussrechnung des Abschlussprüfers im Regelfall erst nach der Aufstellung des Einzel- oder Konzernabschlusses erfolgt (Umlauf 2013, 106). So kann für noch nicht abgerechnete Leistungen des Abschlussprüfers eine Rückstellung im Jahresabschluss gebildet werden (Wulff 2010, 408). Sollte sich die gebildete Rückstellung im Nachhinein als zu hoch oder zu niedrig dotiert erweisen, so ist der Mehr- oder Minderbetrag im Abschluss des Folgegeschäftsjahres anzugeben. Im Fall einer wesentlichen Abweichung sollte eine gesonderte Angabe in Form eines Davon-Vermerks („,davon für das Vorjahr“) erfolgen.

Die im Konzernanhang anzugebenden Honorare sind auf alle vom Konzernabschlussprüfer erbrachten Leistungen anzuwenden, sowohl für das Mutterunternehmen als auch für alle vollkonsolidierten Tochterunternehmen sowie quotal konsolidierte Gemeinschaftsunternehmen (Petersen/Zwirner 2008b, 283; IDW RS HFA 36 2010, Rn. 19; Zülch et al. 2010, 399; Senger/Maier 2013, Rn. 65). Im Fall einer Einbeziehung werden diese von der Angabepflicht befreit, soweit im Konzernabschluss eine Zusammenfassung aller im Konzern angefallenen Angaben erfolgt (Wulff 2010, 409). Dabei nicht auszuweisen sind die Leistungen, welche der Konzernabschlussprüfer bei nicht konsolidierten Tochterunternehmen, Gemeinschaftsunternehmen und assoziierten Unternehmen erbracht hat.

Im Falle eines mehrstufigen Konzerns ist jeder Teilkonzern als Mutterunternehmen eines eigenständigen Konzerns zu behandeln und isoliert zu betrachten. Dabei sind keine Angaben bzgl. über- oder gleichgeordneter Unternehmen erforderlich (Petersen/Zwirner 2008b, 283; IDW RS HFA 36 2010, Rn. 23; Grottel 2014b, Rn. 94). Für den Fall, dass der Abschlussprüfer des Mutter- oder Tochterunternehmens auch gleichzeitig Konzernabschlussprüfer ist, wird im Konzernanhang in der Kategorie „Abschlussprüfung “ zusätzlich das bei der Jahresabschlussprüfung des Mutter- oder Tochterunternehmens angefallene Honorar erfasst. Dazu zählen auch Aufwendungen für die konsolidierungsbedingte Anpassung und Überleitung (Petersen/Zwirner 2008b, 284; Zülch et al. 2010, 399). Ist der Abschlussprüfer eines Tochterunternehmens nicht gleichzeitig Konzernabschlussprüfer und sollte das Honorar trotzdem ohne Vermerk in die Honorarangaben des Konzernabschlusses einfliessen, so wäre die Aussagekraft hinsichtlich der Unabhängigkeit des Konzernprüfers eingeschränkt (Sattler 2011, 119-120). Um dem entgegen zu wirken, schlagen IDW RS HFA 36 (2010) und Grottel (2014b) vor, die Honorarangaben im Konzernanhang als Davon-Vermerk anzugeben.

\section{Bisheriger Forschungsstand}

Die Analyse der Honorarentwicklung sowie der Zusammensetzung der Honorare für Abschlussprüferleistungen auf dem deutschen Prüfungsmarkt ${ }^{5}$ bildet den Gegenstand einiger Untersuchungen. So verwenden Lenz/Bauer (2004) in ihrer fragebogengestützten Studie die Honorardaten von 58 Unternehmen aus den vier Auswahlindizes DAX30, MDAX,

5 Als Beispiele für internationale Studien können die folgenden Studien angeführt werden: Menon/ Williams (2001); Frankel et. al. (2002); Asbaugh et al. (2003); Reynolds et al. (2004); Ciesielski/Weirich (2006); Chan et al. (2012); Baldacchino/Borg (2014). Den meisten dieser Studien ist gemein, dass sie die Abschlussprüferhonorare nur oberflächlich betrachten, da sie lediglich die Datenbasis für den eigentlichen Untersuchungsgegenstand (z.B. die Auswirkung von Beratungshonoraren auf die Qualität der Rechnungslegung) bilden. Lediglich die Studie von Chan et al. (2012) ist mit der hier vorliegenden Studie vergleichbar. Befunde aus den USA sind aufgrund der andersartigen Rahmenbedingungen (z.B. das höhere Haftungsrisiko von US-Abschlussprüfern (Vogt 2009) und die dadurch zu erwartende Risikoprämie im Prüfungshonorar (Seetharaman et al. 2002)) nicht auf den deutschen Prüfungsmarkt übertragbar. 
SDAX und TecDAX für das Jahr 2003. Der Anteil der Honorare für Abschlussprüfungsleistungen an den Gesamthonoraren wird im Durchschnitt über alle Beobachtungen mit $69 \%$ angegeben und das Verhältnis zwischen Beratungs- und Prüfungshonoraren mit $64 \% .^{6}$ Analysen auf Basis der tatsächlichen Honorare sind erst seit der Offenlegungspflicht des $\$ 285 \mathrm{Nr} .17 \mathrm{HGB}$ möglich und wurden erstmals von Lenz et al. (2006), Bigus/ Zimmermann (2008) und Petersen/Zwirner (2008b) vorgelegt.

Bigus/Zimmermann (2008) werten die Honorare von 172 börsennotierten Unternehmen für das Jahr 2005 aus. Sie stellen fest, dass den Beratungshonoraren, die einen Anteil von $41,9 \%$ an den Gesamthonoraren haben, eine ähnliche Bedeutung wie den Prüfungshonoraren zukommt. Für den gleichen Zeitraum weisen Lenz et al. (2006) einen Anteil von $33,76 \%$ der Beratungshonorare an den Gesamthonoraren nach, wobei 12,18\% auf die sonstigen Bestätigungs- und Bewertungsleistungen, 13,77\% auf die sonstigen Leistungen und 7,49\% auf die Steuerberatungsleistungen entfallen. ${ }^{7}$ Der Unterschied in den Ergebnissen der beiden Studien kann auf die unterschiedlichen Stichproben zurückzuführen sein. Für das Jahr 2006 untersuchen Petersen/Zwirner (2008b) die Honorarangaben von 155 Unternehmen aus den vier wichtigen DAX-Indizes. Auch hier zeigt sich, dass die Honorare für Abschlussprüfungsleistungen mit einem Anteil von 69\% am Gesamthonorar überwiegen, gefolgt von den sonstigen Bestätigungs- und Bewertungsleistungen (15\%), den sonstigen Leistungen (10\%) und den Steuerberatungsleistungen (6\%). Allerdings ist die Aussagekraft dieser Studie begrenzt, da die Honorare der weltweiten Netzwerke mit einbezogen werden, weshalb die betrachteten Honorare deutlich über den tatsächlichen Honoraren des Konzernabschlussprüfers i.S.d. $\$ 318$ Abs. 2 HGB liegen dürften. Des Weiteren stellen die Autoren eine sehr heterogene Berichtspraxis bei den Angaben über die Honoraraufwendungen als Bestandteil des Konzernanhangs fest.

In einer mehrperiodischen Untersuchung werten Zülch et al. (2010) die Honorare von 408 kapitalmarktorientierten Unternehmen für die Jahre 2004 bis 2008 aus. Die Ergebnisse zeigen, dass die für Prüfungsleistungen angefallenen Honorare mit einem Durchschnitt von 57,4\% den grössten Teil an den gesamten Honoraren des Betrachtungszeitraums darstellen. Die Honorare für sonstige Bestätigungs- und Bewertungsleistungen weisen indexübergreifend einen Anteil von 26,3\% auf, während der Anteil der Kategorien Steuerberatungsleistungen und sonstige Leistungen als moderat bezeichnet werden kann. Bei einer näheren Analyse der Entwicklung der Honorare für Abschlussprüferleistungen im Zeitverlauf zeigt sich bei dem grössenunabhängigen Massstab „Abschlussprüfungshonorare je 1 Mio. $€$ Bilanzsumme“ ein sukzessiver Rückgang von $27 \%$ über den Betrachtungszeitraum. Darüber hinaus stellen die Verfasser fest, dass 25,2\% der Stichprobenunternehmen mehr Honorare für Nichtprüfungsleistungen als für Prüfungsleistungen verausgaben, was als Indikator für eine mögliche Befangenheit des Abschlussprüfers gilt. Hinsichtlich der Berichterstattungsqualität verweisen die Autoren auf eine positive Entwicklung, wobei immer noch eine grosse Bandbreite an qualitativ unterschiedlichen Angaben vorzufinden ist.

6 Diese Zahlenangaben erscheinen inkonsistent, denn bei einem Anteil der Beratungshonorare an den Gesamthonoraren von 31\% müsste das Verhältnis zwischen Beratungs- zu Prüfungshonoraren bei ca. 45\% liegen. Dieser Widerspruch könnte durch Messunterschiede verursacht sein (Relation aus den aggregierten Honoraren der Stichprobe versus Relation aus Mittelwerten einzelner Honoraranteile). Die in der Publikation enthalten Daten lassen allerdings keinen Rückschluss auf die (vermeintliche) Ursache für diese Inkonsistenz zu.

7 Auch hier tritt eine marginale Inkonsistenz auf, denn die Summe der genannten Honorarkategorien beträgt lediglich $33,44 \%$. 
Ein grundsätzliches Problem besteht insbesondere bei den nicht durchgeführten Abgrenzungen der in den Jahresabschluss einzubeziehenden Prüfungsgesellschaften bzw. -verbünde.

Bei der Analyse von 1.351 Unternehmensjahren für den Zeitraum von 2005 bis 2007 konstatieren Köhler et al. (2010), dass das durchschnittliche Honorar pro Mandant im Jahr 2006 im Vergleich zum Vorjahr um 26 \% gestiegen und im Jahr 2007 im Vergleich zum Vorjahr um 3\% gesunken ist. Bei der Betrachtung des starken Honoraranstieges zu Beginn des Untersuchungszeitraumes sollte berücksichtigt werden, dass sämtliche Unternehmen erstmals in den Anwendungsbereich der IAS-Verordnung gefallen sind, was mit einem erheblichen Mehraufwand im Rahmen der Konzernabschlussprüfung verbunden war. Heß/Stefani (2012) untersuchen die Honorare von 614 kapitalmarktorientierten Unternehmen für das Geschäftsjahr 2010. Die Ergebnisse zeigen, dass die Big 4-Prüfungsgesellschaften durchschnittlich 36\% ihrer Gesamthonorare durch Nichtprüfungsleistungen erzielen, während es bei den übrigen Prüfungsgesellschaften lediglich $18 \%$ sind. Eine mehrperiodische Betrachtung der Honorarzusammensetzung sowie der zeitlichen Entwicklung der Abschlussprüferleistungen ab dem Geschäftsjahr 2009 liegt bislang nicht vor. Eine Analyse der Abschlussprüferhonorare für einen aktuelleren Zeitraum lässt aufgrund von erheblich veränderten Rahmenbedingungen im Vergleich zu früheren Studien andere Ergebnisse erwarten. Die für Abschlussprüfung relevanten Normen wurden weiterentwickelt und erweitert (z.B. BilMoG; diverse Novellen der WPO, u.a. das Berufsaufsichtsreformgesetz; das Grünbuch der EU (Europäische Kommission 2010); die grundlegende Überarbeitung des Code of Ethics der International Federation of Accountants (IFAC), die im Juli 2009 abgeschlossen war (IFAC 2010); Weiterentwicklung der nationalen und internationalen Prüfungsstandards), die Finanzkrise hat sich wesentlich auf die Gestaltung von Abschlussprüferhonoraren ausgewirkt (siehe z.B. Ettredge et al. 2014), es gab zahlreiche Zusammenschlüsse von mittelgrossen WPG (NextTen) und Aufsichtsräte richten häufiger einen Prüfungsausschuss ein. Des Weiteren soll im Folgenden das Verhältnis von Nichtprüfungs- zu Prüfungshonoraren und damit das Ausmass an Unabhängigkeitsgefährdung vor dem Hintergrund der neuen EU-Verordnung eingehend untersucht werden. Solche Analysen leistet die bisherige Forschung ebenfalls nicht.

\section{Stichprobe der empirischen Erhebung}

Die Basis dieser Studie bilden börsennotierte (Mutter-) Unternehmen mit Hauptfirmensitz in Deutschland. Als relevanter Markt wurden die Auswahlindizes DAX30, MDAX, TecDAX und SDAX des Prime Standards der Deutschen Börse ausgewählt. ${ }^{8}$ Hiermit wird ein repräsentativer Querschnitt an deutschen Klein-, Mittelgross- und Grosskonzernen sowie verschiedenen Branchen dargestellt (Zülch et al. 2010, 399). Der Betrachtungszeitraum der Untersuchung erstreckt sich über die Geschäftsjahre 2009 bis 2013. Ausschlaggebend für die Einbeziehung in die im Rahmen dieser Arbeit durchgeführte Untersuchung ist die Indexzugehörigkeit des Unternehmens zum 31.12. eines Jahres. ${ }^{9}$ Unternehmen mit einem vom 31.12. abweichenden Bilanzstichtag werden dem Jahr zugeordnet, in welchem der Bilanzstichtag liegt. Die mit dem BilMoG geänderten Honorarkategorien sind erstma-

8 Zur Abgrenzung der Indizes vgl. Deutsche Börse 2014b, 8-9.

9 Für eine detaillierte Auflistung der historischen Indexzusammensetzung der Equity- und Strategie-Indizes der Deutschen Börse vgl. Deutsche Börse 2014a. 
lig auf Jahres- und Konzernabschlüsse ab dem 1.1.2009 anzuwenden. Folglich stellt eine Untersuchung ab 2009 einen handelsrechtlich gleichbleibenden Rahmen für Abschlussprüferhonorare dar.

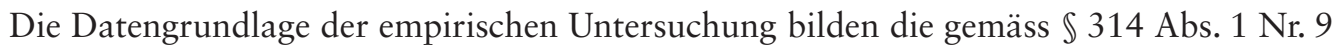
HGB veröffentlichten Abschlussprüferhonorare. So beziehen sich die Honorarangaben stets auf die Honorare, die für den gesetzlich bestellten Abschlussprüfer im Rahmen des Konzernabschlusses für das betreffende Geschäftsjahr angefallen sind. Die Informationen wurden den im Internet bereitgestellten Geschäftsberichten der jeweiligen Konzerne entnommen. Es werden nur die im Inland entrichteten und nicht die weltweit angefallenen Honorare berücksichtigt. Dies geschieht vor dem Hintergrund, dass ausschliesslich der deutsche Prüfungsmarkt untersucht werden soll. Weiterhin sind die Honorarangaben der nachträglich korrigierten und anschliessend veröffentlichten Geschäftsberichte, sofern vorhanden, berücksichtigt. Die für die spätere Untersuchung relevanten Bilanzsummen wurden teilweise den Geschäftsberichten der geprüften Unternehmen, teilweise der Bilanzdatenbank von Hoppenstedt entnommen. Dabei sind nur die (nachträglich korrigierten) Konzernbilanzen und nicht die Einzelbilanzen der verbundenen Unternehmen relevant.

Um einer möglichen Verzerrung der Stichprobe entgegenzuwirken, werden zur Angleichung und Homogenisierung bestimmte Unternehmen aus der Stichprobe eliminiert. Tabelle 1 informiert über die endgültige Stichprobe.

Im ersten Schritt wurden solche Unternehmen aus dem Stichprobenkreis entfernt, die ihren Unternehmenssitz nicht in Deutschland haben. Im nächsten Schritt wurden die Beobachtungswerte ausgesondert, deren Konzernabschluss im Rahmen einer Gemeinschaftsprüfung (Joint Audit) von zwei oder mehr Prüfungsgesellschaften geprüft wurde.

\begin{tabular}{lrrrrrr}
\hline \multicolumn{1}{c}{ Periode } & 2009 & $\mathbf{2 0 1 0}$ & $\mathbf{2 0 1 1}$ & $\mathbf{2 0 1 2}$ & $\mathbf{2 0 1 3}$ & Summe \\
\hline Grundgesamtheit & 160 & 160 & 160 & 160 & 160 & 800 \\
Sitz der Gesellschaft im Ausland & 10 & 9 & 9 & 10 & 10 & 48 \\
Joint Audit & $(2)$ & 2 & $(2)$ & $(2)$ & $(2)$ & 10 \\
Nicht durchgehend in einem der & 32 & 33 & 33 & 32 & 32 & 162 \\
Auswahlindizes & 17 & 17 & 17 & 17 & 17 & 85 \\
Banken, Versicherungen und & 7 & 7 & 7 & 7 & 7 & 35 \\
andere Finanzdienstleister & 92 & 92 & 92 & 92 & 92 & 460 \\
Sonstige Ausschlussgründe & & & & & &
\end{tabular}

Tabelle 1: Auswahl der Stichprobenunternehmen

Um einen Vergleich der Prüfungshonorare über den gesamten Betrachtungszeitraum zu gewährleisten, fand eine Reduzierung der Auswahlstichprobe auf die Unternehmen statt, die sich im gesamten Untersuchungszeitraum 2009 bis 2013 konstant in einem der vier Auswahlindizes befanden. Dadurch werden auch solche Unternehmen berücksichtigt, welche im Untersuchungszeitraum einen Wechsel des Index vollzogen, sofern der Wechsel inner-

10 Es lagen lediglich für das Jahr 2010 zwei Gemeinschaftsprüfungen vor. Um die Homogenität der Stichprobe sicherzustellen, wurden die entsprechenden Beobachtungen auch für die anderen Jahre aussen vor gelassen. 
halb der vier Auswahlindizes stattgefunden hat. Um eine spätere Indexbetrachtung einheitlicher und vergleichbarer zu gestalten, wurden alle Unternehmen dem Index zugeordnet, dem sie im letzten Untersuchungsjahr angehörten. Durch diese Auswahl wird eine konstante Datenbasis mit den selben Unternehmen über den gesamten Betrachtungszeitraum gewährleistet. Weiterhin ermöglicht dieses Vorgehen eine homogene und vergleichbare Betrachtung der Honorare über den Zeitverlauf. Es wurden somit alle Unternehmen ausgeschlossen, die nicht die vollen fünf Untersuchungsjahre im Auswahlindex gelistet waren, sei es z.B. durch ein „Delisting“, eine Insolvenz oder ein „Squeeze-out“.

Für die endgültige Stichprobe sind die Werte von Finanzdienstleistern (Banken, Versicherungen, Immobilien- und Beteiligungsgesellschaften etc.) nicht berücksichtig worden, da diese Unternehmen eine abweichende Bilanzstruktur aufweisen und speziellen prüfungsrechtlichen Vorschriften unterliegen. Bei dieser Vorgehensweise handelt es sich um eine in der internationalen Prüfungsforschung gängige Methode (Lenz/Ostrowski 1999, 399; Bigus/Zimmermann 2008, 163; Umlauf 2013, 194).

Des Weiteren wurden Unternehmen aus der Stichprobe eliminiert, die im Betrachtungszeitraum aufgrund der Umstellung ihres Bilanzstichtages ein Rumpfgeschäftsjahr aufwiesen. Da hier ein verkürztes Geschäftsjahr vorliegt, ist anzunehmen, dass sich dies auch in den Prüfungskosten niederschlägt und somit das Ergebnis der Untersuchung verzerrt (Zülch et al. 2010, 400). Ebenfalls von der Untersuchung ausgeschlossen wurden Unternehmen, für die unzureichende Informationen der im Inland angefallenen Honorare verfügbar waren. So fand bei einigen Unternehmen ausschliesslich eine Angabe der Honorare für Leistungen des internationalen Verbunds statt. Damit war durch einen fehlenden Davon-Vermerk kein Rückschluss auf die im Inland angefallenen Honorare möglich.

Abschliessend wurden aus der Stichprobe Unternehmen ausgeschlossen, welche eine unverhältnismässig grosse Abweichung der Bilanzsumme aufwiesen. ${ }^{11}$ Dies geschah vor dem Hintergrund, dass in der weiteren Untersuchung die Prüfungshonorare mithilfe der Bilanzsumme skaliert werden sollen.

Die finale Stichprobe umfasst 20 Unternehmen aus dem DAX30, 32 Unternehmen aus dem MDAX, 24 Unternehmen aus dem SDAX und 16 Unternehmen aus dem TecDAX. Nach Durchführung der Strichprobenbereinigung verblieben somit insgesamt 460 Beobachtungen, aufgeteilt auf 92 Stichprobenunternehmen für die Untersuchungsperioden 2009 bis 2013.

\section{Untersuchungsergebnisse}

\subsection{Gesamte Stichprobe}

In Tabelle 2 wird ein Überblick über die Grössenordnung der Honorare der einzelnen Geschäftsjahre gegeben, welcher die vier Honorarkategorien sowie das Gesamthonorar enthält. Die dokumentierten Mittelwerte sind stark aggregiert und setzen sich aus sehr heterogenen Einzelbeobachtungen zusammen. Dies wird insbesondere bei der Betrachtung der Minimal- bzw. Maximalwerte der Honorarkategorien deutlich. Die Werte für die Gesamthonorare reichen von 86 TEUR im Minimum im Jahr 2012 bis hin zu 45.000 TEUR im Maximum im Jahr 2009. Die Mediane liegen regelmässig weit unter den arithmetischen

11 Im vorliegenden Fall ist die Bilanzsumme der Deutsche Post AG von 262.964 Mio. EUR in 2008 auf 34.738 Mio. EUR in 2009 gesunken. Diese deutliche Verringerung um über 228.226 Mio. EUR folgte aufgrund der Entkonsolidierung der Deutschen Postbank AG. 
Laudemann et al. | Die zeitliche Entwicklung von Abschlussprüferhonoraren

\begin{tabular}{|c|c|c|c|c|c|c|}
\hline Abschlussprüfungsleistungen & 2009 & 2010 & 2011 & 2012 & 2013 & $\varnothing$ \\
\hline Arithmetisches Mittel & 1.559 & 1.597 & 1.551 & 1.504 & 1.516 & 1.545 \\
\hline Median & 659 & 700 & 715 & 719 & 753 & 709 \\
\hline Standardabweichung & 2.600 & 2.782 & 2.875 & 2.592 & 2.429 & 2.656 \\
\hline Minimum & 79 & 73 & 76 & 68 & 101 & 79 \\
\hline Maximum & 19.000 & 20.000 & 18.000 & 19.000 & 16.000 & 18.400 \\
\hline Jahressumme & 143.457 & 146.948 & 142.738 & 138.375 & 139.512 & 142.206 \\
\hline$\%$-Anteil am Gesamthonorar & $58,4 \%$ & $60,5 \%$ & $60,1 \%$ & $57,5 \%$ & $59,3 \%$ & $59,1 \%$ \\
\hline Andere Bestätigungsleistungen & 2009 & 2010 & 2011 & 2012 & 2013 & $\varnothing$ \\
\hline Arithmetisches Mittel & 632 & 613 & 559 & 670 & 551 & 605 \\
\hline Median & 49 & 68 & 74 & 82 & 73 & 69 \\
\hline Standardabweichung & 2.440 & 2.213 & 2.152 & 2.313 & 1.881 & 2.200 \\
\hline Minimum & 0 & 0 & 0 & 0 & 0 & 0 \\
\hline Maximum & 22.000 & 18.000 & 19.000 & 20.000 & 16.000 & 19.000 \\
\hline Jahressumme & 58.132 & 56.368 & 51.438 & 61.682 & 50.675 & 55.659 \\
\hline$\%$-Anteil am Gesamthonorar & $23,7 \%$ & $23,2 \%$ & $21,6 \%$ & $25,6 \%$ & $21,6 \%$ & $23,2 \%$ \\
\hline Steuerberatungsleistungen & 2009 & 2010 & 2011 & 2012 & 2013 & $\varnothing$ \\
\hline Arithmetisches Mittel & 194 & 152 & 165 & 162 & 171 & 169 \\
\hline Median & 34 & 32 & 26 & 26 & 23 & 28 \\
\hline Standardabweichung & 405 & 357 & 379 & 383 & 376 & 380 \\
\hline Minimum & 0 & 0 & 0 & 0 & 0 & 0 \\
\hline Maximum & 3.000 & 3.000 & 3.000 & 3.000 & 3.000 & 3.000 \\
\hline Jahressumme & 17.892 & 14.019 & 15.163 & 14.874 & 15.772 & 15.544 \\
\hline$\%$-Anteil am Gesamthonorar & $7,3 \%$ & $5,8 \%$ & $6,4 \%$ & $6,2 \%$ & $6,7 \%$ & $6,5 \%$ \\
\hline Sonstige Leistungen & 2009 & 2010 & 2011 & 2012 & 2013 & $\varnothing$ \\
\hline Arithmetisches Mittel & 282 & 278 & 307 & 279 & 318 & 293 \\
\hline Median & 68 & 57 & 55 & 57 & 64 & 60 \\
\hline Standardabweichung & 709 & 669 & 1.080 & 575 & 771 & 761 \\
\hline Minimum & 0 & 0 & 0 & 0 & 0 & 0 \\
\hline Maximum & 5.000 & 5.000 & 10.000 & 4.000 & 5.000 & 5.800 \\
\hline Jahressumme & 25.963 & 25.575 & 28.262 & 25.713 & 29.255 & 26.954 \\
\hline$\%$-Anteil am Gesamthonorar & $10,6 \%$ & $10,5 \%$ & $11,9 \%$ & $10,7 \%$ & $12,4 \%$ & $11,2 \%$ \\
\hline Gesamthonorar des Abschlussprüfers & 2009 & 2010 & 2011 & 2012 & 2013 & $\varnothing$ \\
\hline Arithmetisches Mittel & 2.668 & 2.640 & 2.583 & 2.616 & 2.557 & 2.613 \\
\hline & 1.000 & 1.100 & 1.048 & 1.000 & 1.032 & 1.036 \\
\hline Standardabweichung & 5.391 & 5.276 & 5.477 & 5.165 & 4.528 & 5.167 \\
\hline Minimum & 134 & 128 & 116 & 86 & 146 & 122 \\
\hline Maximum & 45.000 & 41.000 & 39.000 & 41.000 & 32.000 & 39.600 \\
\hline Jahressumme & 245.444 & 242.910 & 237.601 & 240.644 & 235.214 & 240.363 \\
\hline
\end{tabular}

Tabelle 2: Honorare der gesamten Stichprobe in TEUR

Mitteln, was belegt das wenige Unternehmen einen sehr hohen Anteil an den gesamten Abschlussprüferhonoraren zahlen. Ebenfalls deutlich wird dies bei der Betrachtung der einzelnen Leistungskategorien, die von einigen Unternehmen überhaupt nicht nachgefragt 
wurden. Jährlich fielen durchschnittlich 240.363 TEUR für die Leistungen der bestellten Abschlussprüfer an.

In der zeitlichen Entwicklung lässt sich eine leicht rückläufige Streuung der Honorarwerte erkennen. Deutlicher hingegen ist der Rückgang der Maximalwerte der Honorare für Abschlussprüfungsleistungen und andere Bestätigungsleistungen. Da sich die Honorare für Steuerberatungsleistungen und sonstige Leistungen auf konstantem Niveau bewegen, sinkt auch der Maximalwert der Gesamthonorare des Abschlussprüfers im Zeitverlauf spürbar. Auffallend sind die hohen Gesamthonorare für das Jahr 2009 (245.444 TEUR), die in den folgenden Jahren nach und nach auf einen absoluten Wert von 235.214 TEUR (2013) fallen. Die Honorare für andere Bestätigungsleistungen verhalten sich grundsätzlich vergleichbar, wenngleich das Jahr 2012 mit einem Gesamtwert von 61.682 TEUR einen kurzen Ausreisser darstellt. Die Honorare für Abschlussprüfungsleistungen schwanken geringfügig im Zeitverlauf und weisen eine leicht rückläufige Tendenz auf. Während für Steuerberatungsleistungen keine eindeutige zeitliche Entwicklungsrichtung zu erkennen ist, lässt sich für die sonstigen Leistungen eine Tendenz zu steigenden Honoraren konstatieren.

Die aggregierten Honorare für Prüfungsleistungen stellen mit einem durchschnittlichen Anteil von 59,1\% die betragsmässig bedeutsamste Honorarkategorie dar. Mit deutlichem Abstand folgen die Honorare für andere Bestätigungsleistung $(23,2 \%)$ und für sonstige Leistungen (11,2\%). Die Steuerberatungsleistungen machen mit 6,5\% den geringsten Anteil am Gesamthonorar aus. Insgesamt sind nur wenige Veränderungen im Zeitverlauf bei den Honoraranteilen zu beobachten. Es fällt allerdings auf, dass die Bedeutung der Honorare für sonstige Leistungen im Zeitablauf zunimmt, was zu Lasten der Honorare für andere Bestätigungsleistung geht.

Die Betrachtung der absoluten Werte hat ergeben, dass sie einer starken Streuung unterliegen. Dies dürfte vor allem daran liegen, dass es sich um Unternehmen verschiedenster Branchen und Grössen handelt. Um die Abschlussprüfungshonorare weiter beurteilen zu können, wird in Abbildung 1 die Kennzahl „Abschlussprüfungshonorar je Quadratwurzel aus 1 Mio. $€$ Bilanzsumme“ betrachtet. Eine Skalierung mithilfe der Bilanzsumme ist deshalb sinnvoll, weil Honorarstudien durchgehend aufzeigen, dass die Grösse des Mandanten, zumeist an der Bilanzsumme gemessen, der wichtigste Treiber der Höhe der Abschlussprüfungshonorare ist (Simunic 1980; Eichenseher/Danos 1981; Quick et al. 1998, 781; Bigus/Zimmermann 2008, 168; Köhler et al. 2010, 9). Zudem ist darauf zu verweisen, dass sich Abschlussprüfungshonorare idealerweise aus einer Zeitgebühr und einer Wertgebühr, die sich an der Bilanzsumme des geprüften Unternehmens orientiert, zusammensetzen sollen (IDW 2012, A.720). Der Mittelwert der skalierten Abschlussprüfungshonorare sinkt von 18,65 im Jahr 2009 auf 17,33 im Jahr 2013. Der Verlauf findet jedoch nicht linear statt. So sinkt die Kennzahl von ihrem Maximum in Höhe von 18,65 im Jahr 2009 auf das Minimum von 16,72 im Jahr 2011, um anschliessend wieder anzusteigen. Die in Abbildung 1 dokumentierte Trendlinie zeigt eine im Zeitverlauf rückläufige Entwicklung. Aus den Ergebnissen von Scheffé-Mittelwertvergleichstests lässt sich jedoch schliessen, dass der rückläufige Trend nicht signifikant ist, denn sämtliche Mittelwertdifferenzen erweisen sich als nicht signifikant (Signifikanzniveau zwischen 0,775 und 1,000). 
Die zur Skalierung genutzten Bilanzsummen der Gesamtstichprobe steigen hingegen jährlich an. ${ }^{12}$

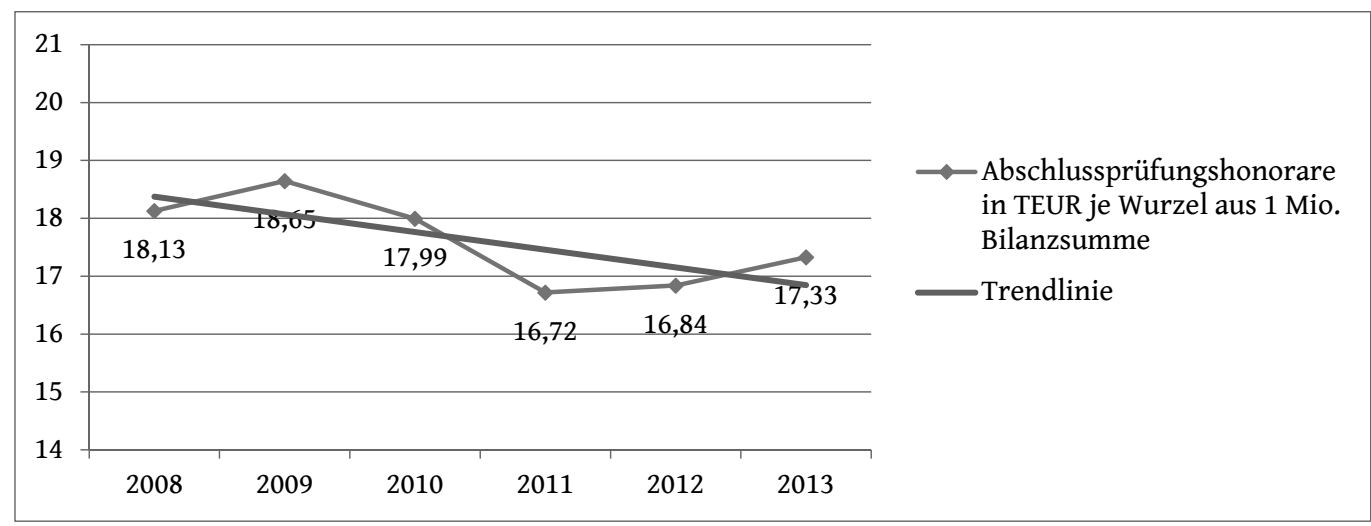

Abbildung 1: Prüfungshonorar je Quadratwurzel aus 1 Mio. $€$ Bilanzsumme der gesamten Stichprobe

Im Hinblick auf die Unabhängigkeit des Abschlussprüfers ist u.a. der Anteil des Gesamthonorars von Interesse, welcher nicht auf die Abschlussprüfung entfällt. Dieser ist vergleichsweise konstant, liegt zwischen 39,5\% (2010) und 42,5\% (2012) und weist keinen eindeutigen Trend auf. Betrachtet man das Verhältnis von Nichtprüfungs- zu Prüfungsleistungshonoraren, so liegt dieses im Beobachtungszeitraum zwischen 65,3\% (2010) und 73,9\% (2012). Vor dem Hintergrund der neuen EU-Verordnung erscheint dieser Anteil bedenklich, denn diese begrenzt die Honorare für Nichtprüfungsleistungen auf maximal 70\% des Durchschnitts der in den letzten drei aufeinanderfolgenden Geschäftsjahren gezahlten Honorars für Abschlussprüfungsleistungen. Sofern man diese Kennzahlen zunächst für jedes Stichprobenunternehmen einzeln berechnet und erst dann einen Durchschnitt bildet, zeigt sich, dass kleinere Unternehmen weniger Nichtprüfungsleistungen nachfragen als grössere Unternehmen; denn die Relationen verschieben sich zugunsten der Honorare für Abschlussprüfungsleistungen, d.h. das Verhältnis der Honorare für Nichtprüfungsleistungen zu den Honoraren für Abschlussprüferleistungen liegt zwischen 57\% (2011 und 2012) und 63\% (2009), wobei t-Tests keine signifikanten Unterschiede zwischen den einzelnen Jahren anzeigen.

Um die Werte weiter interpretieren zu können, ist es sinnvoll, sich zusätzlich die Anzahl der Unternehmen anzuschauen, welche überhaupt Beratungsleistungen in Anspruch genommen haben. Wäre diese Zahl sehr niedrig, würde dies bedeuten, dass die Beratungsleistungen mit hohem Volumen auf wenige Unternehmen konzentriert wären. Im vorliegenden Fall nehmen jedoch nahezu alle Stichprobenunternehmen Beratungsleistungen vom bestellten Abschlussprüfer entgegen. Dabei ist ein geringer Rückgang von 90 Unternehmen im Jahr 2009 auf 88 Unternehmen im Jahr 2013 zu erkennen. Vergleicht man weiterhin die Anzahl der Unternehmen, bei denen der Honoraranteil der Nichtprüfungsleistungen

12 Verwendet man zur Skalierung statt der Bilanzsumme die Umsatzerlöse als alternatives Grössenmerkmal, so zeigen sich gleichgerichtete Ergebnisse. Diese Aussage gilt auch für die nachfolgenden indexspezifischen Analysen. 
die Grenze von 70\% überschreitet, wird deutlich, dass durchschnittlich 32,4\% der Unternehmen über diesem Grenzwert liegen.

\subsection{Abschlussprüferhonorare nach Auswahlindizes}

\subsubsection{DAX30}

Mit einem durchschnittlichen Anteil von 65,6\% an den Gesamthonoraren der gesamten Stichprobe entfällt der grösste Anteil der Honorare auf den DAX. Tabelle 3 gibt einen Überblick über die Bandbreite, in der sich die absoluten Honorardaten der DAX30-Unternehmen bewegen. Die Gesamthonorare der betrachteten Unternehmen liegen im Mittel der fünf Jahre zwischen 980 TEUR und 39.600 TEUR. Das niedrigste Gesamthonorare liegt bei 900 TEUR (für die Jahre 2012 und 2013), welches von einem Unternehmen mit einer Bilanzsumme von 6.597 Mio. EUR offengelegt wurde (K+S AG). Im Gegensatz hierzu wurde das höchste Gesamthonorar von 45.000 TEUR mit einer korrespondierenden Bilanzsumme von 152.636 Mio. EUR im Jahr 2009 ausgewiesen (E.ON SE). Es wird deutlich, dass die Honorare im DAX30 stark voneinander abweichen.

Den Honoraren der E.ON SE kommt über den gesamten Betrachtungszeitraum eine Sonderstellung zu, denn deren Gesamthonorare stellen stets das Maximum dar. Gleichzeitig wiesen auch die Honorare für andere Bestätigungsleistungen der E.ON SE die mit deutlichem Abstand höchsten Werte dieser Kategorie auf (Ø 19.000 TEUR). Diese repräsentieren im Mittel einen Anteil von 45,4\% der für diese Kategorie aufsummierten Honorare. Es ist jedoch zu beobachten, dass die absoluten Honorarwerte der E.ON SE im Zeitverlauf deutlich zurückgehen. So reduzieren sich die Gesamthonorare bzw. die Honorare für andere Bestätigungsleistungen von 45.000 TEUR bzw. 22.000 TEUR im Jahr 2009 auf 32.000 TEUR bzw. 16.000 TEUR im Jahr 2013. Dies bedeutet einen Rückgang von 28,9\% bzw. $27,3 \%$ über den Zeitraum von fünf Jahren. Auch für die Honorarkategorie Abschlussprüfungsleistung weist die E.ON SE in allen fünf Jahren das Maximum auf, obwohl die Volkswagen AG gemessen an der Bilanzsumme das jeweils grösste DAX30-Unternehmen ist. Eine Begründung für die vergleichsweise hohen Honorare lässt sich in den Geschäftsberichten nicht finden. ${ }^{13}$

Bei Betrachtung der absoluten Gesamthonorare zeigt sich eine deutlich rückläufige Tendenz, was sich in den geringsten Honoraren für das Jahr 2013 (147.585 TEUR) zeigt. Die gleiche Entwicklung lässt sich bei den Abschlussprüfungshonoraren erkennen. So fallen die absoluten Honorare für Prüfungsleistungen im Zeitverlauf von 2009 bis 2013 um durchschnittlich 2,0\%. Besonders hoch fällt der Rückgang der Prüfungshonorare von 2011 auf 2012 aus. Dafür verantwortlich sind insbesondere die Unternehmen Volkswagen AG (-5 Mio. €), Thyssen Krupp (-2 Mio. €), Bayer AG (-2 Mio. €) und RWE AG (-1,5 Mio. $€)$. Da bei diesen Unternehmen im Jahr 2012 kein Prüferwechsel stattfand, lässt sich diese rückläufige Entwicklung nicht mit einem damit zusammenhängenden Fee Cutting-Effekt (vgl. hierzu Simon/Francis 1988; Ettredge/Greenberg 1990; Woo/Kob 2001; DeFond et al. 2002; Kallunki et al. 2007; Tate 2007) erklären. Er dürfte viel mehr belegen, dass im DAX30 trotz einer duopolistischen Anbieterstruktur der Wettbewerb funktioniert. Dagegen

13 Die E.ON SE nennt in ihren Geschäftsberichten zwar die von dem Abschlussprüfer durchgeführten Tätigkeiten, jedoch immer im gleichen Wortlaut. Man könnte vermuten, dass es sich um eine standardisierte Auflistung der möglichen durchgeführten Tätigkeiten des Abschlussprüfers handelt und nicht um eine Erklärung für die tatsächlich im Geschäftsjahr angefallenen Leistungen. 
Laudemann et al. | Die zeitliche Entwicklung von Abschlussprüferhonoraren

\begin{tabular}{|c|c|c|c|c|c|c|}
\hline Abschlussprüfungsleistungen & 2009 & 2010 & 2011 & 2012 & 2013 & $\varnothing$ \\
\hline Arithmetisches Mittel & 4.615 & 4.773 & 4.692 & 4.336 & 4.160 & 4.515 \\
\hline Median & 3.400 & 3.200 & 2.950 & 3.000 & 2.950 & 3.100 \\
\hline Standardabweichung & 4.287 & 4.713 & 5.018 & 4.474 & 4.091 & 4.517 \\
\hline Minimum & 817 & 800 & 800 & 700 & 700 & 763 \\
\hline Maximum & 19.000 & 20.000 & 18.000 & 19.000 & 16.000 & 18.400 \\
\hline Jahressumme & 92.302 & 95.469 & 93.835 & 86.719 & 83.206 & 90.306 \\
\hline$\%$-Anteil am Gesamthonorar & $56,8 \%$ & $59,6 \%$ & $58,8 \%$ & $54,8 \%$ & $56,4 \%$ & $57,3 \%$ \\
\hline Andere Bestätigungsleistungen & 2009 & 2010 & 2011 & 2012 & 2013 & $\varnothing$ \\
\hline Arithmetisches Mittel & 2.278 & 2.257 & 1.999 & 2.569 & 1.995 & 2.220 \\
\hline Median & 663 & 423 & 583 & 1.000 & 900 & 714 \\
\hline Standardabweichung & 4.926 & 4.413 & 4.362 & 4.531 & 3.729 & 4.392 \\
\hline Minimum & 0 & 0 & 0 & 0 & 0 & 0 \\
\hline Maximum & 22.000 & 18.000 & 19.000 & 20.000 & 16.000 & 19.000 \\
\hline Jahressumme & 45.553 & 45.139 & 39.971 & 51.374 & 39.908 & 44.389 \\
\hline$\%$-Anteil am Gesamthonorar & $28,0 \%$ & $28,1 \%$ & $25,0 \%$ & $32,4 \%$ & $27,0 \%$ & $28,1 \%$ \\
\hline Steuerberatungsleistungen & 2009 & 2010 & 2011 & 2012 & 2013 & $\varnothing$ \\
\hline Arithmetisches Mittel & 380 & 279 & 375 & 327 & 269 & 326 \\
\hline Median & 84 & 44 & 97 & 50 & 5 & \\
\hline Standardabweichung & 726 & 680 & 712 & 699 & 673 & 698 \\
\hline Minimum & 0 & 0 & 0 & 0 & 0 & 0 \\
\hline Maximum & 3.000 & 3.000 & 3.000 & 3.000 & 3.000 & 3.000 \\
\hline Jahressumme & 7.608 & 5.587 & 7.493 & 6.537 & 5.375 & 6.520 \\
\hline$\%$-Anteil am Gesamthonorar & $4,7 \%$ & $3,5 \%$ & $4,7 \%$ & $4,1 \%$ & $3,7 \%$ & $4,1 \%$ \\
\hline Sonstige Leistungen & 2009 & 2010 & 2011 & 2012 & 2013 & $\varnothing$ \\
\hline Arithmetisches Mittel & 852 & 703 & 920 & 687 & 955 & 823 \\
\hline Median & 188 & 200 & 350 & 211 & 300 & 250 \\
\hline Standardabweichung & 1.356 & 1.239 & 2.178 & 992 & 1.447 & 1.442 \\
\hline Minimum & 0 & 0 & 0 & 0 & 0 & 0 \\
\hline Maximum & 5.000 & 5.000 & 10.000 & 4.000 & 5.000 & 5.800 \\
\hline Jahressumme & 17.044 & 14.066 & 18.405 & 13.731 & 19.096 & 16.468 \\
\hline$\%$-Anteil am Gesamthonorar & $10,5 \%$ & $8,8 \%$ & $11,5 \%$ & $8,7 \%$ & $12,9 \%$ & $10,5 \%$ \\
\hline Gesamthonorar des Abschlussprüfers & 2009 & 2010 & 2011 & 2012 & 2013 & $\varnothing$ \\
\hline Arithmetisches Mittel & 8.125 & 8.013 & 7.985 & 7.918 & 7.379 & 7.884 \\
\hline Median & 5.000 & 4.950 & 4.700 & 4.950 & 4.650 & 4.850 \\
\hline Standardabweichung & 9.695 & 9.525 & 10.046 & 9.261 & 7.859 & 9.277 \\
\hline Minimum & 999 & 1.100 & 1.000 & 900 & 900 & 980 \\
\hline Maximum & 45.000 & 41.000 & 39.000 & 41.000 & 32.000 & 39.600 \\
\hline Jahressumme & 162.507 & 160.261 & 159.704 & 158.361 & 147.585 & 157.684 \\
\hline
\end{tabular}

Tabelle 3: Deskriptive Auswertung der Honorare des DAX30 in TEUR

verzeichnen die Honorare der Kategorie für sonstige Leistungen einen durchschnittlich jährlichen Anstieg von 2,1\%, der jedoch nicht als kontinuierlich bezeichnet werden kann. Die Honorare für Steuerberatungsleistungen, die aufgrund des geringen Volumens von nachrangiger Bedeutung sind, weisen eine sinkende Tendenz auf. Bei den anderen Bestäti- 
gungsleistungen ist bei grundsätzlich fallendem Verlauf ein einmaliger Anstieg für das Jahr 2012 zu erkennen. ${ }^{14}$ Die angeführten Entwicklungen finden sich teilweise auch in der relativen Honorarzusammensetzung wieder. Der relative Anteil der Prüfungshonorare sinkt marginal von 56,8\% (Jahr 2009) auf 56,4\% (Jahr 2013). Gleiches gilt für den Anteil der anderen Bestätigungsleistungen (28\% vs. $27 \%$ ). Dagegen steigt der Anteil der sonstigen Leistungen im gleichen Zeitraum von 10,5\% auf 12,9\%. Die Relevanz der Honorare für Steuerberatungsleistungen ist ebenfalls rückläufig.

Die Betrachtung der grössenunabhängigen Kennzahl „Abschlussprüfungshonorar je Quadratwurzel aus 1 Mio. € Bilanzsumme“ in Abbildung 2 bringt weitere Erkenntnisse.

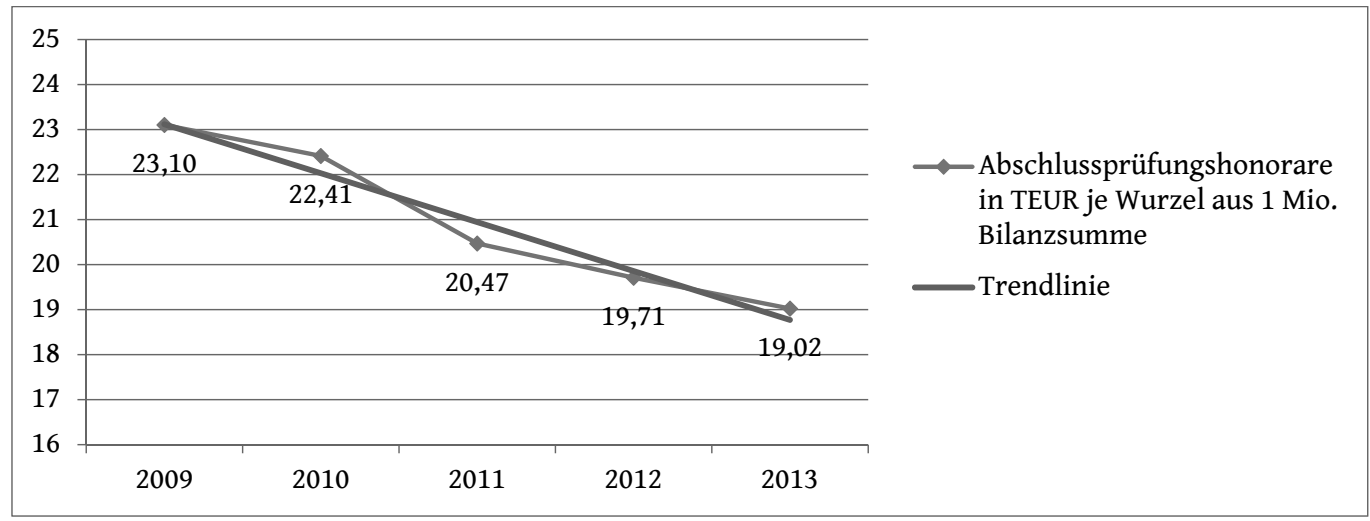

Abbildung 2: Prüfungshonorar je Quadratwurzel aus 1 Mio. $€$ Bilanzsumme des DAX30

Das durchschnittliche Niveau des grössenbereinigten Prüfungshonorars der DAX30-Unternehmen liegt bei einem Wert von 20,94. Es wird deutlich, dass auch die Entwicklung des relativen Abschlussprüfungshonorars rückläufig ist, was auch aus dem Verlauf der Trendlinie ersichtlich wird. So geht sein Mittelwert von 23,10 im Jahr 2009 auf den Wert von 19,02 im Jahr 2013 zurück. Dies stellt einen Rückgang des relativen Honorars von 17,7\% innerhalb von fünf Jahren dar und reflektiert die stärkste Entwicklung im Vergleich aller betrachteten Indizes. Diese Entwicklung kann auf den immer noch auf dem Markt herrschenden Wettbewerb der WPG zurückzuführen sein (Köhler et al. 2010, 22; Zülch et al. 2010, 401-402). Die im Zeitablauf rückläufige Entwicklung der relativen Abschlussprüfungshonorare muss aber basierend auf Scheffé-Mittelwertvergleichstests als nicht signifikant bezeichnet werden (Signifikanzniveau zwischen 0,840 und 1,000).

Für die Beurteilung der Unabhängigkeit des Abschlussprüfers ist es hilfreich, den Anteil der Nicht-Abschlussprüfungshonorare am Gesamthonorar zu betrachten. Dieser ist leicht gestiegen und er beträgt im Durchschnitt 42,7\%, d.h. das Verhältnis der Nichtprüfungshonorare zu den Prüfungshonoraren beträgt im Mittel knapp 75\% und liegt somit über der von der EU formulierten kritischen Grenze. Sofern diese Kennzahl zunächst für alle Stich-

14 Für diesen Anstieg zeichnen sich vor allem folgende Unternehmen verantwortlich: Bayer AG (+3 Mio. $€)$, Continental AG (+1 Mio. €), Fresenius SE \& Co. KGaA(+3 Mio. €), SAP AG (+2 Mio. €) und Volkswagen AG (+1 Mio. €). Lediglich im Geschäftsbericht der Continental AG findet sich diesbezüglich eine Erklärung und die zusätzlichen Honorare für andere Bestätigungsleistungen lassen sich auf eine Anleiheemission zurückführen. 
probenunternehmen einzeln ermittelt und anschliessend ein Durchschnitt gebildet wird, beträgt das Verhältnis zwischen den Nichtprüfungshonoraren und den Prüfungshonoraren lediglich $67,7 \%$. Wie t-Tests belegen, unterscheiden sich die Beobachtungen der einzelnen Jahre nicht signifikant voneinander. Erneut zeigt sich also, dass die Nachfrage nach Nichtprüfungsleistungen auch innerhalb des DAX30 mit zunehmender Grösse des Mandanten steigt. Die Anzahl der Unternehmen, deren Honoraranteil der Nichtprüfungsleistungen die Grenze von $70 \%$ überschreitet, liegt bei durchschnittlich $41 \%$. Dabei nahmen nicht alle der 20 Unternehmen in den Jahren 2009, 2010 und 2011 Beratungsleistungen vom bestellten Abschlussprüfer in Anspruch, wobei sich in 2009 und 2010 ein Unternehmen und im Jahr 2011 zwei Unternehmen auf die Durchführung der Abschlussprüfungsleistung beschränkten.

\subsubsection{MDAX}

Die Ergebnisse für die Honorarentwicklung im MDAX sind in Tabelle 4 aufgeführt. Über den Betrachtungszeitraum hinweg wurde ein durchschnittliches Gesamthonorar von 50.177 TEUR gezahlt, welches $20,9 \%$ des indexübergreifenden Gesamthonorars ausmacht. Somit entfällt auf den MDAX der zweitgrösste, jedoch im Vergleich zum DAX30 erheblich geringere Anteil an den insgesamt angefallenen Abschlussprüferhonoraren. Dagegen repräsentieren die 32 Unternehmen mit einem Anteil von 34,8\% den grössten Teil der Stichprobe.

Die Beträge des Gesamthonorars reichen im Durchschnitt der fünf Jahre von 263 TEUR bis zu 5.832 TEUR. Im Vergleich zum DAX30 ist deren Spannweite, trotz einer höheren Anzahl an Messwerten, wesentlich geringer. Das geringste Gesamthonorar beträgt 170 TEUR, ist im Jahr 2011 angefallen und betrifft die Fielmann AG. Der Maximalbetrag liegt bei 7.146 TEUR und ist im Jahr 2013 bei der GEA Group AG angefallen. Die dazugehörigen Bilanzsummen belaufen sich auf 727 Mio. EUR (Fielmann AG) und 6.263 Mio. EUR (GEA Group AG). Eine auffallende Entwicklung in der Kategorie der Abschlussprüfungshonorare zeigt sich bei der GEA Group AG. So übersteigt der Wert von 4.901 TEUR für das Jahr 2013 die in den Vorjahren angefallenen Honorare des Unternehmens zumindest um das Doppelte, obwohl es zu keinen nennenswerten Veränderungen der Bilanzsumme bzw. der Umsatzerlöse kam. Darüber hinaus stellt dieser Wert das im Betrachtungszeitraum höchste Abschlussprüfungshonorar des Index dar. ${ }^{15}$

Eine Betrachtung der absoluten Honorare zeigt deutliche Schwankungen. So sind für 2011 die niedrigsten Gesamthonorare (46.730 TEUR) und für 2013 die höchsten Gesamthonorare (52.965 TEUR) gezahlt worden. Ein eindeutiger Trend ist nicht zu erkennen. Die Honorare für Abschlussprüfungsleistungen und für Steuerberatungsleistungen entwickeln sich vergleichbar. Der starke Anstieg der Honorare für Steuerberatungsleistungen im Jahr 2013 ist primär auf die GEA Group zurückzuführen (+911 TEUR), ohne dass der Geschäftsbericht erkennen lässt, um welche Steuerberatungsleistungen es sich konkret handelt. Bei den Honoraren für andere Bestätigungsleistungen ist dagegen eine rückläufige Entwicklung ersichtlich. Sie tragen im Jahr 2009 mit 9.396 TEUR massgeblich zum hohen

15 Der Geschäftsbericht der GEA Group für das Jahr 2013 gibt keinen Aufschluss über die Gründe der deutlich erhöhten Abschlussprüfungshonorare. 


\begin{tabular}{|c|c|c|c|c|c|c|}
\hline Abschlussprüfungsleistungen & 2009 & 2010 & 2011 & 2012 & 2013 & $\varnothing$ \\
\hline Arithmetisches Mittel & 1.019 & 985 & 925 & 989 & 1.075 & 999 \\
\hline Median & 800 & 791 & 758 & 733 & 778 & 772 \\
\hline Standardabweichung & 761 & 719 & 689 & 764 & 1.005 & 788 \\
\hline Minimum & 207 & 230 & 170 & 176 & 199 & 196 \\
\hline Maximum & 3.142 & 3.009 & 2.800 & 2.872 & 4.901 & 3.345 \\
\hline Jahressumme & 32.594 & 31.526 & 29.589 & 31.638 & 34.407 & 31.951 \\
\hline$\%$-Anteil am Gesamthonorar & $62,3 \%$ & $64,6 \%$ & $63,3 \%$ & $63,2 \%$ & $65,0 \%$ & $63,7 \%$ \\
\hline Andere Bestätigungsleistungen & 2009 & 2010 & 2011 & 2012 & 2013 & $\varnothing$ \\
\hline Arithmetisches Mittel & 294 & 217 & 251 & 220 & 229 & 242 \\
\hline Median & 94 & 100 & 114 & 100 & 100 & 102 \\
\hline Standardabweichung & 552 & 370 & 430 & 322 & 314 & 398 \\
\hline Minimum & 0 & 0 & 0 & 0 & 0 & 0 \\
\hline Maximum & 1.987 & 1.900 & 2.200 & 1.400 & 1.200 & 1.737 \\
\hline Jahressumme & 9.396 & 6.939 & 8.035 & 7.046 & 7.315 & 7.746 \\
\hline$\%$-Anteil am Gesamthonorar & $18,0 \%$ & $14,2 \%$ & $17,2 \%$ & $14,1 \%$ & $13,8 \%$ & $15,5 \%$ \\
\hline Steuerberatungsleistungen & 2009 & 2010 & 2011 & 2012 & 2013 & $\varnothing$ \\
\hline Arithmetisches Mittel & 190 & 161 & 129 & 115 & 179 & 155 \\
\hline Median & 77 & 100 & 47 & 46 & 45 & 63 \\
\hline Standardabweichung & 287 & 210 & 160 & 138 & 234 & 206 \\
\hline Minimum & 0 & 0 & 0 & 0 & 0 & 0 \\
\hline Maximum & 1.192 & 921 & 500 & 412 & 954 & 796 \\
\hline Jahressumme & 6.091 & 5.149 & 4.126 & 3.667 & 5.735 & 4.954 \\
\hline$\%$-Anteil am Gesamthonorar & $11,6 \%$ & $10,5 \%$ & $8,8 \%$ & $7,3 \%$ & $10,8 \%$ & $9,8 \%$ \\
\hline Sonstige Leistungen & 2009 & 2010 & 2011 & 2012 & 2013 & $\varnothing$ \\
\hline Arithmetisches Mittel & 132 & 163 & 156 & 241 & 172 & 173 \\
\hline Median & 71 & 88 & 40 & 47 & 62 & 62 \\
\hline Standardabweichung & 165 & 257 & 277 & 409 & 269 & 275 \\
\hline Minimum & 0 & 0 & 0 & 0 & 0 & 0 \\
\hline Maximum & 700 & 1.400 & 1.100 & 2.000 & 1.261 & 1.292 \\
\hline Jahressumme & 4.217 & 5.204 & 4.980 & 7.724 & 5.508 & 5.527 \\
\hline$\%$-Anteil am Gesamthonorar & $8,1 \%$ & $10,7 \%$ & $10,7 \%$ & $15,4 \%$ & $10,4 \%$ & $11,0 \%$ \\
\hline Gesamthonorar des Abschlussprüfers & 2009 & 2010 & 2011 & 2012 & 2013 & $\varnothing$ \\
\hline Arithmetisches Mittel & 1.634 & 1.526 & 1.460 & 1.565 & 1.655 & 1.568 \\
\hline Median & 1.100 & 1.131 & 1.100 & 1.110 & 1.075 & 1.103 \\
\hline Standardabweichung & 1.490 & 1.195 & 1.170 & 1.271 & 1.497 & 1.325 \\
\hline Minimum & 339 & 399 & 170 & 199 & 208 & 263 \\
\hline Maximum & 6.514 & 5.300 & 5.200 & 5.000 & 7.146 & 5.832 \\
\hline Jahressumme & 52.298 & 48.818 & 46.730 & 50.075 & 52.965 & 50.177 \\
\hline
\end{tabular}

Tabelle 4: Deskriptive Auswertung der Honorare des MDAX in TEUR 
Gesamthonorar des Jahres bei. ${ }^{16}$ Hinsichtlich der Honorare für sonstige Leistungen lässt sich hingegen von einer steigenden Tendenz sprechen. Bei der relativen Zusammensetzung der Honorarkategorien lassen sich Schwankungen bzw. Tendenzen erkennen. So geht das Honorar für sonstige Leistungen im Jahr 2012 mit 15,4\% deutlich über den Durchschnitt von $11,1 \%$ hinaus. ${ }^{17}$ Der Honoraranteil für Abschlussprüfungsleistungen nimmt tendenziell zu, der für andere Bestätigungsleistungen dagegen ab. Die relative Bedeutung der Steuerberatungsleistung verläuft grundsätzlich rückläufig, wenngleich der Honoraranteil im Jahr 2013 wieder zunimmt.

Anhand der Kennzahl „Abschlussprüfungshonorar je Quadratwurzel aus 1 Mio. € Bilanzsumme" wird in Abbildung 3 der zeitliche Verlauf des grössenbereinigten Honorars dargestellt. Das Niveau des Honorars liegt mit einem Wert von durchschnittlich 17,89 unter dem der DAX30-Honorare. Nach einem anfänglichen Rückgang des Wertes von 2009 $(19,76)$ auf 16,68 im Jahr 2011 steigt dieser daraufhin wieder leicht an. Dennoch weist die Trendlinie einen leicht fallenden Verlauf auf, für den jedoch keine Signifikanz feststellbar ist (Signifikanzniveau der Scheffé-Mittelwertvergleichstests zwischen 0,845 und 1,000).

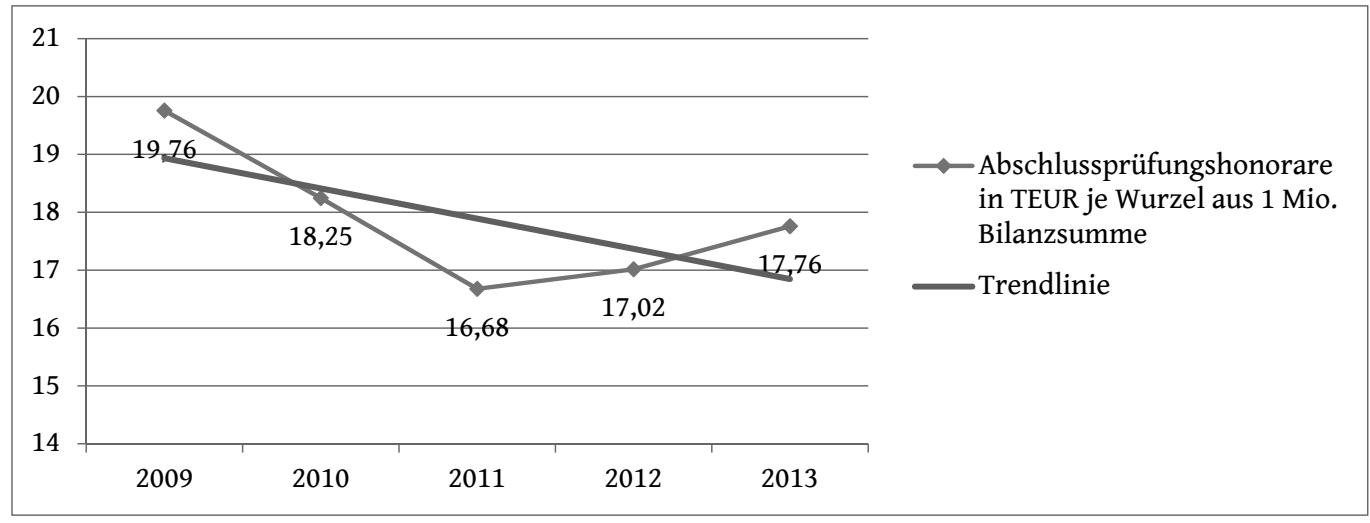

Abbildung 3: Prüfungshonorar je Quadratwurzel aus 1 Mio. $€$ Bilanzsumme des MDAX

Der Anteil der Nicht-Abschlussprüfungshonorare am Gesamthonorar liegt bei 36,3\% und ist leicht rückläufig, d.h. das Verhältnis der Nichtprüfungshonorare zu den Prüfungshonoraren liegt im Durchschnitt bei $57 \%$ und ist damit geringer als bei den DAX30-Unternehmen. Sofern diese Kennzahl zunächst für alle Stichprobenunternehmen einzeln ermittelt und anschliessend ein Durchschnitt gebildet wird, beträgt das Verhältnis zwischen den Nichtprüfungshonoraren und den Prüfungshonoraren 56,8\%. Dies spricht dafür, dass die Nachfrage nach Nichtprüfungsleistungen im MDAX wenig grössenabhängig ist. Dieser Durchschnittswert liegt zudem unterhalb der von der EU als kritisch angesehenen Grenze und weist gemäss den Ergebnissen von t-Tests im Zeitablauf keine signifikant verschiedenartigen Höhen auf. Ferner liegt er signifikant unter dem des DAX30 ( $T=1,737 ; \mathrm{p}=0,084)$.

16 Der Wert in 2009 wird durch die einmalig erhöhten Honorare für andere Bestätigungsleistungen der Klöckner \& Co SE (1.987 TEUR) und RHÖN-KLINIKUM AG (1.748 TEUR) verursacht. Die Werte übersteigen in diesem Jahr den Periodendurchschnitt um das Dreifache. Grund dafür war für beide Unternehmen eine Kapitalerhöhung.

17 Für diesen Anstieg zeichnen sich vor allem folgende Unternehmen verantwortlich: Bilfinger SE, Celesio AG und Salzgitter AG. 
Wiederum empfiehlt es sich, für die Interpretation dieser Werte die Anzahl der Unternehmen zu betrachten, welche Beratungsleistungen in Anspruch genommen haben. In den ersten zwei Jahren, d.h. 2009 und 2010, fragten alle 32 Unternehmen Beratungsleistungen nach. Für die Jahre 2011 und 2012 hingegen nur 30 und 31 für das Jahr 2013. Betrachtet man weiterhin die Anzahl der Unternehmen, bei denen der Honoraranteil der Nichtprüfungsleistungen die Grenze von 70\% überschreitet, zeigt sich, dass 32,5\% der Unternehmen aus dem MDAX über dieser Schwelle liegen.

\subsubsection{SDAX}

Im Folgenden werden die Honorare der 24 Unternehmen aus dem SDAX für den Zeitraum von 2009 bis 2013 betrachtet. Der Anteil des durchschnittlichen Gesamthonorars des SDAX am Gesamthonorar der gesamten Stichprobe beträgt lediglich 7,9\%, obwohl die 24 Unternehmen einen Anteil von 26,1\% aller betrachteten Unternehmen ausmachen. Das durchschnittliche Gesamthonorar aller bestellten Abschlussprüfer der einbezogenen SDAX-Unternehmen beträgt 19.016 TEUR. Damit liegt dieser Wert deutlich unter denen des DAX30 und MDAX.

Informationen zur Zusammensetzung der Honorare liefert Tabelle 5. Die gezahlten Gesamthonorare der Unternehmen reichen von durchschnittlich 122 TEUR bis hin zu 2.902 TEUR. Das niedrigste Gesamthonorar beträgt 86 TEUR, ist im Jahr 2012 angefallen und wurde von einem Unternehmen mit einer Bilanzsumme von 1.092 Mio. EUR (KWS SAAT AG) gezahlt. Dagegen wurde das höchste Gesamthonorar im Jahr 2010 in Höhe von 3.249 TEUR für ein Unternehmen mit einer Bilanzsumme von 2.879 Mio. EUR (Heidelberger Druckmaschinen AG) berechnet.

Die absoluten Gesamthonorare unterliegen einer unregelmässigen Schwankung. Im Jahr 2009 sind die geringsten Honorare an den Abschlussprüfer gezahlt worden (17.574 TEUR). Dagegen steigen die Gesamthonorare im nachfolgenden Geschäftsjahr (2010) auf den höchsten Wert (20.631 TEUR). Das Jahr 2010 stellt mit seinen hohen Honoraren für sonstige Leistungen eine Ausnahme dar. Die Unternehmen Heidelberger Druckmaschinen AG und Koenig \& Bauer AG mit Honoraren von 2.413 TEUR bzw. 1.000 TEUR prägen massgeblich diesen Ausreisser. Die Geschäftsberichte beider Unternehmen enthalten keinen Hinweis auf den Grund der Abweichung. ${ }^{18}$ Die absoluten Honorare für Abschlussprüfungsleistungen steigen im Zeitverlauf von 10.298 TEUR (2009) auf 12.387 TEUR (2013). Auch bei den Honoraren für andere Bestätigungsleistungen ist ein ansteigender Trend zu konstatieren. Auffallend ist vor allem, dass sich die Honorare für andere Bestätigungsleistungen im Jahr 2012 im Vergleich zum Vorjahr um ca. 30\% erhöht haben. Dieser deutliche Anstieg ist auf die Vossloh AG (+500 TEUR) zurückzuführen, ohne dass deren Geschäftsbericht Aufschluss über die Art dieser Leistung gibt. Die Honorare für Steuerberatungsleistungen schwanken im Zeitablauf stark. In Bezug auf die Honoraranteile lässt sich eine steigende Bedeutung der Abschlussprüfungsleistung und der anderen Bestätigungsleistung erkennen. Der Honoraranteil für Steuerberatungsleistungen sinkt im Jahr 2010 im Vergleich zum Vorjahr stark ab und verbleibt dann auf einem in etwa konstanten

18 Beide Unternehmen geben in ihrem Geschäftsbericht lediglich den Betrag der Honorare an. So weisen aber beide Unternehmen in ihren Geschäftsberichten auf die schwierige Lage nach der Wirtschaftskrise und das deutlich geschrumpfte Weltmarktvolumen hin, welches mit einer Neuausrichtung kompensiert werden soll. Beide Unternehmen entstammen der gleichen Branche (Spezialmaschinenbau/ Druckmaschinen). 
Laudemann et al. | Die zeitliche Entwicklung von Abschlussprüferhonoraren

\begin{tabular}{|c|c|c|c|c|c|c|}
\hline Abschlussprüfungsleistungen & 2009 & 2010 & 2011 & 2012 & 2013 & $\varnothing$ \\
\hline Arithmetisches Mittel & 429 & 481 & 452 & 479 & 516 & 471 \\
\hline Median & 401 & 391 & 346 & 386 & 426 & 390 \\
\hline Standardabweichung & 307 & 353 & 293 & 341 & 318 & 322 \\
\hline Minimum & 79 & 73 & 76 & 68 & 101 & 79 \\
\hline Maximum & 1.585 & 1.690 & 1.245 & 1.406 & 1.320 & 1.449 \\
\hline Jahressumme & 10.298 & 11.537 & 10.854 & 11.503 & 12.387 & 11.316 \\
\hline$\%$-Anteil am Gesamthonorar & $58,6 \%$ & $55,9 \%$ & $59,5 \%$ & $62,9 \%$ & $60,9 \%$ & $59,6 \%$ \\
\hline Andere Bestätigungsleistungen & 2009 & 2010 & 2011 & 2012 & 2013 & $\varnothing$ \\
\hline Arithmetisches Mittel & 77 & 72 & 70 & 91 & 97 & 81 \\
\hline Median & 25 & 32 & 11 & 32 & 16 & 23 \\
\hline Standardabweichung & 117 & 102 & 112 & 185 & 200 & 143 \\
\hline Minimum & 0 & 0 & 0 & 0 & 0 & 0 \\
\hline Maximum & 400 & 409 & 400 & 900 & 900 & 602 \\
\hline Jahressumme & 1.836 & 1.726 & 1.683 & 2.186 & 2.320 & 1.950 \\
\hline$\%$-Anteil am Gesamthonorar & $10,5 \%$ & $8,4 \%$ & $9,2 \%$ & $12,0 \%$ & $11,4 \%$ & $10,3 \%$ \\
\hline Steuerberatungsleistungen & 2009 & 2010 & 2011 & 2012 & 2013 & $\varnothing$ \\
\hline Arithmetisches Mittel & 125 & 96 & 82 & 92 & 108 & 101 \\
\hline Median & 13 & 18 & 17 & 8 & 13 & 14 \\
\hline Standardabweichung & 221 & 175 & 162 & 180 & 213 & 190 \\
\hline Minimum & 0 & 0 & 0 & 0 & 0 & 0 \\
\hline Maximum & 700 & 700 & 700 & 800 & 800 & 740 \\
\hline Jahressumme & 2.992 & 2.293 & 1.960 & 2.204 & 2.603 & 2.410 \\
\hline$\%$-Anteil am Gesamthonorar & $17,0 \%$ & $11,1 \%$ & $10,8 \%$ & $12,0 \%$ & $12,8 \%$ & $12,7 \%$ \\
\hline Sonstige Leistungen & 2009 & 2010 & 2011 & 2012 & 2013 & $\varnothing$ \\
\hline Arithmetisches Mittel & 102 & 211 & 156 & 100 & 126 & 139 \\
\hline Median & 37 & 55 & 52 & 69 & 59 & 54 \\
\hline Standardabweichung & 152 & 440 & 404 & 156 & 206 & 272 \\
\hline Minimum & 0 & 0 & 0 & 0 & 0 & 0 \\
\hline Maximum & 692 & 2.004 & 1.985 & 742 & 833 & 1.251 \\
\hline Jahressumme & 2.448 & 5.075 & 3.745 & 2.399 & 3.031 & 3.340 \\
\hline$\%$-Anteil am Gesamthonorar & $13,9 \%$ & $24,6 \%$ & $20,5 \%$ & $13,1 \%$ & $14,9 \%$ & $17,4 \%$ \\
\hline Gesamthonorar des Abschlussprüfers & 2009 & 2010 & 2011 & 2012 & 2013 & $\varnothing$ \\
\hline Arithmetisches Mittel & 732 & 860 & 760 & 762 & 848 & 792 \\
\hline Median & 592 & 634 & 626 & 486 & 582 & 584 \\
\hline Standardabweichung & 524 & 741 & 686 & 671 & 705 & 665 \\
\hline Minimum & 134 & 128 & 116 & 86 & 146 & 122 \\
\hline Maximum & 2.253 & 3.249 & 3.107 & 2.900 & 3.000 & 2.902 \\
\hline Jahressumme & 17.574 & 20.631 & 18.242 & 18.292 & 20.341 & 19.016 \\
\hline
\end{tabular}

Tabelle 5: Deskriptive Auswertung der Honorare des SDAX in TEUR

Niveau. Für die sonstigen Leistungen lässt sich eine starke Schwankung ihres Honoraranteils im Zeitverlauf identifizieren.

Die Entwicklung der Kennzahl „Abschlussprüfungshonorar je Quadratwurzel aus 1 Mio. $€$ Bilanzsumme" kann der Abbildung 4 entnommen werden. Die grössenbereinig- 
ten Abschlussprüfungshonorare zeigen für die Jahre 2009 bis 2013 bei nur geringfügig ansteigenden Bilanzsummen keine signifikanten Änderungen auf, was auch durch den Verlauf der Trendlinie verdeutlich wird. Scheffé-Mittelwertvergleichstests belegen, dass zwischen den Beobachtungen der einzelnen Jahre keine signifikanten Unterschiede bestehen (Signifikanzniveau zwischen 0,987 und 1,000). Die Werte streuen recht eng um den Mittelwert von 13,36. Dieser stellt im Vergleich zu den anderen Indizes den niedrigsten Wert dar. Der leichte Anstieg der skalierten Honorare von 2009 (13,49) auf 2010 (13,92) wird durch einen niedrigeren Wert in 2011 (12,72) unterbrochen, welcher bis 2013 wieder 13,8 und damit in etwa das alte Niveau erreicht. Das konstante Niveau lässt für die Unternehmen des SDAX keinen veränderten Wettbewerbsdruck unter den WPG vermuten.

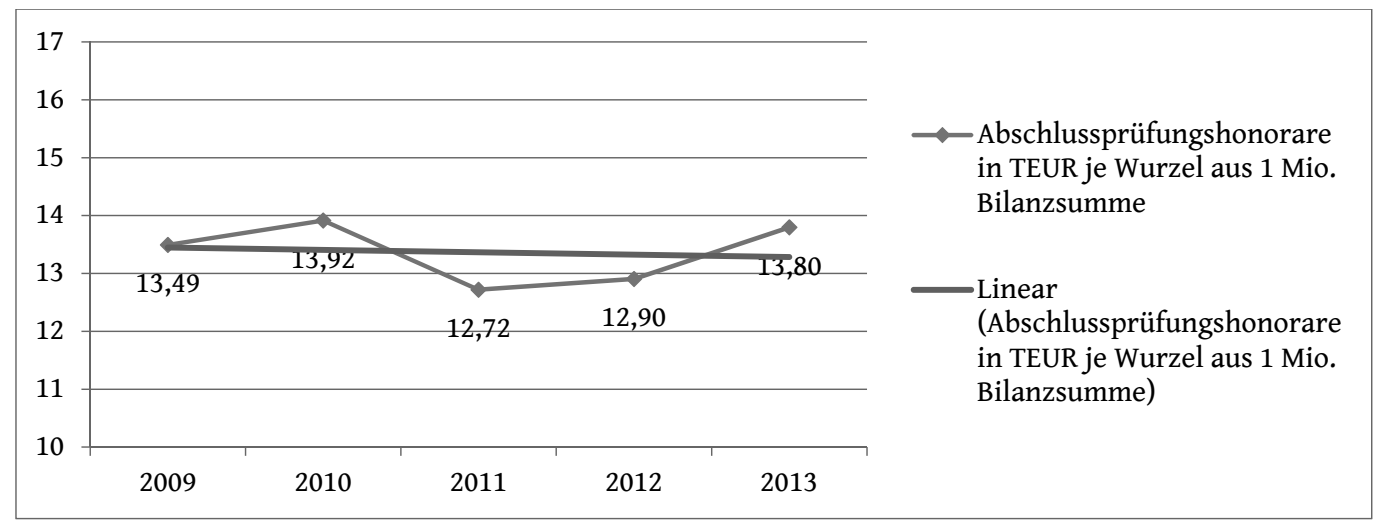

Abbildung 4: Prüfungshonorar je Quadratwurzel aus 1 Mio. $€$ Bilanzsumme der SDAX

Betrachtet man den Anteil der Nicht-Prüfungshonorare am Gesamthonorar, so ist dieser leicht gestiegen und er beträgt im Durchschnitt 40,4\%, sodass das Verhältnis der Nichtprüfungshonorare zu den Prüfungshonoraren im Mittel bei 67,8\% und damit in einem kritischen Bereich liegt. Bei alternativer Berechnung dieser Kennzahl ergibt sich ein etwas niedrigerer Wert von 63,7\%, wobei sich hier im Zeitablauf eine stark rückläufige Tendenz zeigt. Daraus lässt sich schliessen, dass sich in den jüngsten Jahren vor allem kleinere Unternehmen des SDAX hinsichtlich der Nachfrage nach Nichtprüfungsleistungen stärker zurückgehalten haben. Diese Kennzahl für den SDAX unterscheidet sich nicht signifikant von der des DAX30 (T=-0,512; $\mathrm{p}=0,609)$ und des MDAX ( $\mathrm{T}=1,132 ; \mathrm{p}=0,259)$. Der Anteil der Nicht-Prüfungshonorare kann zudem im Untersuchungszeitraum als relativ stabil bezeichnet werden, denn t-Tests legen keine signifikanten Unterschiede zwischen den einzelnen Jahren offen.

Untersucht man die Anzahl der Unternehmen, die Beratungsleistungen in den einbezogenen Geschäftsjahren durch den bestellten Abschlussprüfer in Anspruch genommen haben, so verringert sich diese kaum (von 23 im Jahr 2009 auf 22 im Jahr 2013). Dies lässt den Rückschluss zu, dass der Anteil der Beratungshonorare (alternative Berechnung) tatsächlich im Laufe der betrachteten fünf Perioden weniger wurde und die Zahlen nicht nur dadurch zustande kommen, dass weniger Unternehmen Beratungsleistungen nachgefragt haben. Kritisch anzumerken ist, dass der Honoraranteil der Nichtprüfungsleistungen von 
30,8 \% der Unternehmensbeobachtungen aus dem SDAX über dem Grenzwert von 70\% liegen.

\subsubsection{TecDAX}

Die Teilstichprobe des TecDAX enthält Honorardaten von 16 Unternehmen. Diese stellen mit einem durchschnittlichen Gesamthonorar von 13.486 TEUR, was einen Anteil von $5,6 \%$ des indexübergreifenden Gesamthonorars ausmacht, und einem Anteil an allen Stichprobenunternehmen von $17,4 \%$ den auf die Honorare bezogenen kleinsten Index der Gesamtstichprobe dar.

Tabelle 6 gibt einen Überblick über die Honorardaten. Aufgrund der geringen Anzahl an untersuchten Unternehmen des TecDAX ist es möglich, dass einzelne Unternehmen mit grossen Abweichungen in den Honoraren einen massgeblichen Einfluss ausüben. Die Honorardaten des Index weisen jedoch diesbezüglich keine Auffälligkeiten auf. Im Betrachtungszeitraum werden im Mittel Gesamthonorare von 191 TEUR bis 2.618 TEUR gezahlt. Das niedrigste Gesamthonorar berichtet die CANCOM SE im Jahr 2012 mit 159 TEUR (bei einer Bilanzsumme von 209 TEUR). Das höchste Gesamthonorar erzielte der Abschlussprüfer der freenet AG mit 3.109 TUER im Jahr 2009 (bei einer Bilanzsumme von 3.034 TEUR).

Das arithmetische Mittel der Honorare für Abschlussprüfungsleistungen steigt kontinuierlich an. Diese Entwicklung spiegelt sich grundsätzlich auch in den Mittelwerten der Gesamthonorare und der Honorare für Steuerberatungsleistungen wider. Bei der Betrachtung der Honorare für Steuerberatungsleistungen ist darauf hinzuweisen, dass ein Grossteil auf einige wenige Unternehmen konzentriert ist. So weisen von den 80 zur Verfügung stehenden Honorardaten ${ }^{19} 59$ (73,8\%) ein Steuerberatungshonorar von weniger als 100 TEUR auf. $^{20}$ Die Honorare für Steuerberatungsleistungen haben sich von 2010 auf 2011 um 60\% und von 2011 auf 2012 um knapp 56\% erhöht. Diese Veränderungen sind durch einen starken Anstieg der Nachfrage nach Steuerberatungsleistungen durch die United Internet AG verursacht. (2011: +563 TEUR und 2012: + 528 TEUR). Deren Geschäftsberichte liefern keinen Aufschluss über die Art der nachgefragten Steuerberatungsleistungen. In Bezug auf die Honorare für sonstige Leistungen lässt sich kein eindeutiger Trend feststellen. Als Ausreisser kann der Maximalwert von 1.374 TEUR für andere Bestätigungsleistungen im Geschäftsjahr 2010 identifiziert werden. Hierbei entfallen über die Hälfte der im Geschäftsjahr für diese Kategorie angefallenen Honorare auf nur ein Unternehmen. Dabei handelt es sich um einmalig gezahlte Honorare, welche einem besonderen Umstand im Geschäftsjahr geschuldet sind. ${ }^{21}$

Betrachtet man die relative Zusammensetzung der Honorarkategorien, spiegelt sich die jährliche Steigung der Honorare für Abschlussprüfungsleistungen in dieser partiell wider. So liegt der Anteil dieser Honorare im Durchschnitt bei 64,0\%. Der prozentuale Anteil der Honorare für die anderen Bestätigungsleistungen schwankt, deutet aber eher auf eine rückläufige Tendenz hin. Das relative Honorar für Steuerberatungsleistungen entspricht dem zuvor aufgezeigten Trend. Bei den Honoraren für sonstige Leistungen ist keine klare

1916 Unternehmen in einem Zeitraum von 5 Jahren.

20 Hierbei wurde bei 38 Werten kein Honorar festgestellt.

21 Von den gezahlten Honoraren der Drägerwerk AG \& Co. KGaA in Höhe von 1.374 TEUR fällt ein Betrag in Höhe von 1.051 TEUR im Zusammenhang mit einer Kapitalerhöhung und einer Einzelfallversicherung zur Haftungshöchstgrenze an. 


\begin{tabular}{|c|c|c|c|c|c|c|}
\hline Abschlussprüfungsleistungen & 2009 & 2010 & 2011 & 2012 & 2013 & $\varnothing$ \\
\hline Arithmetisches Mittel & 516 & 526 & 529 & 532 & 595 & 540 \\
\hline Median & 488 & 505 & 448 & 428 & 459 & 466 \\
\hline Standardabweichung & 331 & 306 & 303 & 292 & 323 & 311 \\
\hline Minimum & 142 & 148 & 170 & 153 & 143 & 151 \\
\hline Maximum & 1.173 & 1.118 & 1.068 & 1.064 & 1.157 & 1.116 \\
\hline Jahressumme & 8.263 & 8.416 & 8.461 & 8.515 & 9.512 & 8.633 \\
\hline$\%$-Anteil am Gesamthonorar & $63,2 \%$ & $63,8 \%$ & $65,5 \%$ & $61,2 \%$ & $66,4 \%$ & $64,0 \%$ \\
\hline Andere Bestätigungsleistungen & 2009 & 2010 & 2011 & 2012 & 2013 & $\varnothing$ \\
\hline Arithmetisches Mittel & 84 & 160 & 109 & 67 & 71 & 98 \\
\hline Median & 0 & 16 & 20 & 32 & 30 & 17 \\
\hline Standardabweichung & 232 & 362 & 191 & 124 & 123 & 206 \\
\hline Minimum & 0 & 0 & 0 & 0 & 0 & 0 \\
\hline Maximum & 919 & 1.374 & 636 & 493 & 488 & 782 \\
\hline Jahressumme & 1.347 & 2.564 & 1.749 & 1.076 & 1.133 & 1.574 \\
\hline$\%$-Anteil am Gesamthonorar & $10,3 \%$ & $19,4 \%$ & $13,5 \%$ & $7,7 \%$ & $7,9 \%$ & $11,8 \%$ \\
\hline Steuerberatungsleistungen & 2009 & 2010 & 2011 & 2012 & 2013 & $\varnothing$ \\
\hline Arithmetisches Mittel & 75 & 62 & 99 & 154 & 129 & 104 \\
\hline Median & 29 & 6 & 15 & 11 & 19 & 16 \\
\hline Standardabweichung & 127 & 108 & 237 & 363 & 286 & 224 \\
\hline Minimum & 0 & 0 & 0 & 0 & 0 & 0 \\
\hline Maximum & 499 & 383 & 946 & 1.474 & 1.162 & 893 \\
\hline Jahressumme & 1.201 & 990 & 1.584 & 2.466 & 2.059 & 1.660 \\
\hline$\%$-Anteil am Gesamthonorar & $9,2 \%$ & $7,5 \%$ & $12,2 \%$ & $17,7 \%$ & $14,4 \%$ & $12,2 \%$ \\
\hline Sonstige Leistungen & 2009 & 2010 & 2011 & 2012 & 2013 & $\varnothing$ \\
\hline Arithmetisches Mittel & 141 & 77 & 71 & 116 & 101 & 101 \\
\hline Median & 39 & 27 & 12 & 15 & 42 & 27 \\
\hline Standardabweichung & 282 & 135 & 94 & 274 & 207 & 198 \\
\hline Minimum & 0 & 0 & 0 & 0 & 0 & 0 \\
\hline Maximum & 986 & 523 & 301 & 1.098 & 838 & 749 \\
\hline Jahressumme & 2.254 & 1.230 & 1.132 & 1.859 & 1.620 & 1.619 \\
\hline$\%$-Anteil am Gesamthonorar & $17,3 \%$ & $9,3 \%$ & $8,8 \%$ & $13,4 \%$ & $11,3 \%$ & $12,0 \%$ \\
\hline Gesamthonorar des Abschlussprüfers & 2009 & 2010 & 2011 & 2012 & 2013 & $\varnothing$ \\
\hline Arithmetisches Mittel & 817 & 825 & 808 & 870 & 895 & 843 \\
\hline Median & 537 & 661 & 597 & 630 & 876 & 660 \\
\hline Standardabweichung & 791 & 626 & 589 & 750 & 613 & 674 \\
\hline Minimum & 188 & 161 & 193 & 159 & 254 & 191 \\
\hline Maximum & 3.109 & 2.302 & 2.315 & 2.798 & 2.566 & 2.618 \\
\hline Jahressumme & 13.065 & 13.200 & 12.925 & 13.916 & 14.324 & 13.486 \\
\hline
\end{tabular}

Tabelle 6: Deskriptive Auswertung der Honorare des TecDAX in TEUR

Entwicklung identifizierbar. Erkennbar ist der grosse Anteil von 19,4\% der Honorare für andere Bestätigungsleistungen in 2010, welcher von den zuvor identifizierten Honorarabweichungen der Drägerwerk AG \& Co. KGaA verursacht wird. 
Die Kennzahl „Abschlussprüfungshonorar je Quadratwurzel aus 1 Mio. € Bilanzsumme“ weist über den Fünfjahreszeitraum nur eine geringe Veränderung auf (vgl. Abbildung 5). Die grössenbereinigten Werte streuen in einem geringen Masse um das Mittel von 18,6. Abgesehen von der ersten Betrachtungsperiode, steigen die relativen Honorare vom kleinsten Wert in $2010(18,1)$ zum grössten Wert in $2013(19,6)$ an. Dabei ist das Niveau der Werte mit dem des MDAX vergleichbar und es liegt somit über dem des SDAX. Obwohl die Unternehmen des TecDAX grössenmässig denen des SDAX am ähnlichsten sind, scheint hier ein Branchenaufschlag für technologieintensive Unternehmen vorzuliegen. Zusammenfassend kann ein schwach steigender Trend der grössenbereinigten Kennzahl identifiziert werden, was auch aus dem Verlauf der Trendlinie in Abbildung 5 hervorgeht. Dieser Anstieg ist allerdings statistisch nicht signifikant, denn die Resultate der Scheffé-Mittelwertvergleichstests zeigen keine signifikanten Unterschiede zwischen den Beobachtungen der einzelnen Jahre an (Signifikanzniveau zwischen 0,993 und 1,000).

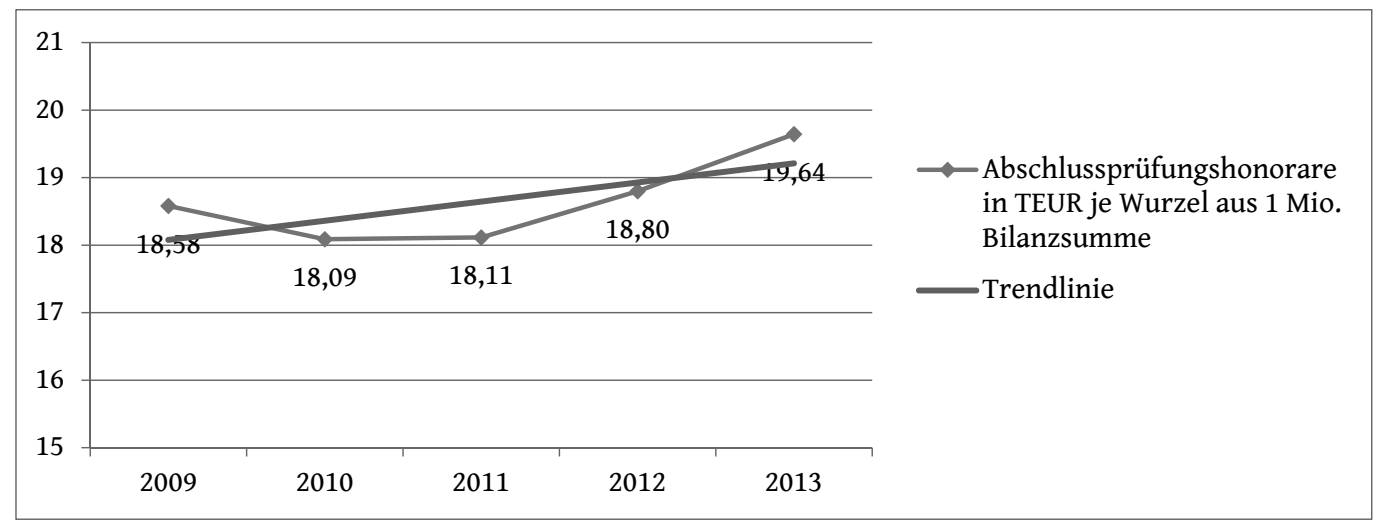

Abbildung 5: Prüfungshonorar je Quadratwurzel aus 1 Mio. € Bilanzsumme des TecDAX

Die Unternehmen des TecDAX weisen im Durschnitt einen Anteil der Nichtprüfungshonorare an den Gesamthonoraren von 36,0\% auf. Dies entspricht einem Anteil der Nichtprüfungshonorare an den Prüfungshonoraren von 56,3\%, was deutlich unter der von der EU formulierten Höchstgrenze liegt. Bei alternativer Berechnung dieser Kennzahl liegt dieses Verhältnis sogar nur bei $47,8 \%$, woraus sich schliessen lässt, dass grössere Unternehmen des TecDAX im vermehrten Masse Beratungsleistungen bei ihrem Abschlussprüfer nachfragen. Auch hier lassen sich anhand von t-Tests keine signifikanten Unterschiede in der Ausprägung dieser Kennzahl für die einzelnen Jahre erkennen. Der Anteil der Nichtprüfungshonorare ist im TecDAX signifikant geringer als der des DAX30 (T=-2,546; $\mathrm{p}=0,012)$ und des SDAX ( $\mathrm{T}=-2,013$; $\mathrm{p}=0,046)$, während im Vergleich zum MDAX keine signifikanten Unterschiede zu konstatieren sind ( $\mathrm{T}=-1,349 ; \mathrm{p}=0,179)$. Die Anzahl der Unternehmen, welche Beratungsleistungen in Anspruch genommen haben, schwankt zwischen 15 und den maximal möglichen 16 Unternehmen. Vergleicht man weiterhin die Anzahl der Unternehmen, deren Honoraranteil der Nichtprüfungsleistungen die Grenze von 70\% überschreiten, wird deutlich, dass 23,8 \% der im TecDAX vertretenen Unternehmen über diesem Grenzwert liegen. 


\section{Zusammenfassung}

Die vorliegende Studie hat sich mit der Untersuchung der Abschlussprüferhonorare von Unternehmen der Börsenindizes DAX30, MDAX, SDAX und TecDAX beschäftigt. Ihr Ziel war es, die Daten der Jahre 2009 bis 2013 zu erheben, zu beschreiben und zu analysieren. So sollten neben der Analyse der absoluten Werte auch die Verhältnisse sowie die relativen Werte der Honorare mithilfe von Kennzahlen abgebildet werden.

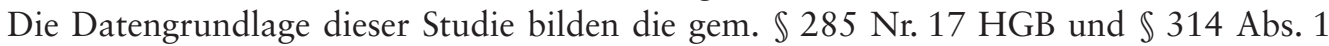
Nr. 9 HGB im Anhang eines Jahresabschlusses veröffentlichten Honorardaten. Für die Veröffentlichung schreibt der Gesetzgeber einen Ausweis, getrennt nach den Kategorien Abschlussprüfung, andere Bestätigungsleistungen, Steuerberatungsleistungen und sonstige Leistungen, vor. Auf Grundlage dieser Einteilung wurde der Fokus der Untersuchung auf die zeitliche Entwicklung der Abschlussprüferhonorare im Gesamtkontext und auf die einzelnen Auswahlindizes gelegt.

Die empirischen Befunde zeigen, dass mit durchschnittlich 59,1\% die Honorare für Abschlussprüfungsleistungen in den Jahren 2009 bis 2013 überwiegen. In Bezug auf die Nichtprüfungsleistungen kommt den durchschnittlichen Honoraren für andere Bestätigungsleistungen mit 23,2\% die grösste Bedeutung zu. Es folgen die Honorare für sonstige Leistungen $(11,2 \%)$ und für Steuerberatungsleistungen $(6,5 \%)$. Die Gesamthonorare des Abschlussprüfers weisen im Zeitverlauf eine leicht fallende Tendenz auf, was in erster Linie durch sinkende Honorare für Abschlussprüfungsleistungen und andere Bestätigungsleistungen verursacht ist. Die grössenbereinigte Kennzahl „Abschlussprüfungshonorare je Quadratwurzel aus 1 Mio. € Bilanzsumme“ sinkt leicht von 18,65 in 2009 auf 17,33 in 2013. Das Verhältnis von Abschlussprüfungsleistungen zu Gesamthonorar und das Verhältnis von Beratungs- zu Abschlussprüfungsleistungen bleiben im Zeitverlauf bei 59,1\% bzw. 69,2\% konstant. Der von der EU beschlossene kritische Wert für das Verhältnis von Beratungs- zu Prüfungshonoraren in Höhe von $70 \%$, der als Indikator für die mögliche Befangenheit des Abschlussprüfers zu sehen ist, wird in 32,4\% der Fälle überschritten.

Im nächsten Schritt wurde aufgrund der sehr heterogenen Grundgesamtheit die indexspezifische Entwicklung der Honorare untersucht. Die Analyse des DAX30 ergibt eine deutlich rückläufige Tendenz in Bezug auf die Gesamthonorare und die Honorare für Abschlussprüfungsleistungen. Diese Entwicklung spiegelt sich auch bei den durchschnittlichen grössenbereinigten Abschlussprüfungshonoraren wider, die einen deutlichen Rückgang von 23,10 in 2009 auf 19,02 in 2013 zeigen, wenngleich Mittelwertvergleichstests keine Signifikanz anzeigen. Diese Entwicklung bei den Prüfungshonoraren lässt vermuten, dass trotz eines Anbieterduopols (KPMG und $\mathrm{PwC}$ ) der Preiswettbewerb funktioniert. Dieser Preiswettbewerb dürfte massgeblich aus den hohen Reputationsgewinnen, die sich aus der Betreuung von DAX30-Mandanten ergeben, resultieren. Diesem Rückgang bei den Honoraren für Abschlussprüferleistungen steht insbesondere ein Anstieg bei den Honoraren für sonstige Leistungen gegenüber. Insgesamt steigt damit im Untersuchungszeitraum auch das Verhältnis von Beratungs- zu Prüfungshonoraren. Diese Relation beträgt im Durchschnitt $75 \%$, überschreitet somit die als kritisch angesehen Grenze und dürfte deshalb geeignet sein, Besorgnis der Befangenheit auszulösen.

Die Untersuchung des MDAX weist für die Gesamthonorare sowie für die Honorare für Abschlussprüfungsleistungen und für Steuerberatungsleistungen starke Schwankungen auf, ohne dass ein eindeutiger Trend zu erkennen ist. Dagegen verlaufen die Honorare für andere Bestätigungsleistungen rückläufig und die für sonstige Leistungen steigend. Weiterhin 
weisen die skalierten Abschlussprüfungshonorare einen rückläufigen Trend auf. So gehen diese von 2009 bis 2013 von 19,76 auf 17,76 zurück, wobei Scheffé-Tests diese Entwicklung als nicht signifikant klassifizieren. Dagegen bleiben die Verhältnisse von Beratungszu Gesamt- und Prüfungshonoraren relativ konstant bei 36,3\% bzw. 58,1\%. Das Verhältnis der Nichtprüfungshonorare zu den Prüfungshonoraren liegt im Durchschnitt bei $57 \%$ und damit weit unter der Relation für den DAX30.

Die Honorare im SDAX sind durch deutliche Schwankungen gekennzeichnet. Einige Ausreisser verzerren die Ergebnisse. Allerdings steigen die Honorare für Abschlussprüfungsleistungen und andere Bestätigungsleistungen im Zeitablauf. Das durchschnittliche skalierte Prüfungshonorar liegt auf dem indexweit niedrigsten Niveau von 13,32 und lässt keinen Trend erkennen. Dagegen geht der mögliche Indikator für die Abhängigkeit des Prüfers in Form des Verhältnisses von Beratungs- zu Prüfungsleistungen im Zeitablauf auf $64,2 \%$ zurück.

Das jährlich steigende Gesamthonorar der TecDAX-Unternehmen spiegelt sich auch im Anstieg der Honorare für Abschlussprüfungsleistungen und Steuerberatungsleistungen wider. Die durch die Bilanzsumme skalierten Honorare zeigen, entgegen den anderen Indizes, einen insignifikanten minimal steigenden Trend auf und liegen erheblich über denen des SDAX. Dagegen beträgt der Anteil der Nichtprüfungshonorare an den Prüfungshonoraren im Durchschnitt 56,3\% und ist somit mit dem des SDAX vergleichbar.

Der hohe Anteil der Honorare für Nichtprüfungsleistungen ist bedenklich, denn er liegt, insbesondere bei den DAX30-Unternehmen, in vielen Fällen über der von der EU kritisch angesehenen Grenze von 70\% des Honorars für Abschlussprüfungsleistungen. Dies deutet auf eine Gefährdung der Unabhängigkeit hin und geht mit der Gefahr entsprechender Negativwahrnehmung der Kapitalmärkte einher, was über ein höheres wahrgenommenes Risiko zu höheren Kapitalkosten führen könnte. Diesbezüglich besteht sowohl auf Seiten der Mandanten als auf Seiten der Abschlussprüfer Handlungsbedarf im Sinne einer Verringerung der ökonomischen Abhängigkeit des Abschlussprüfers.

Für die Beurteilung der vorgestellten Ergebnisse ist zu beachten, dass diese sich lediglich auf einen Teilmarkt des deutschen Prüfungsmarktes beziehen und die aufgezeigten Entwicklungstendenzen und Relationen folglich auch nur für diesen Teilmarkt sowie den betrachteten Zeitraum gelten. Künftige Studien könnten sich somit zum einen mit den Honoraren sämtlicher börsennotierter Unternehmen beschäftigen und zum anderen insbesondere Honorare von nichtbörsennotierten Unternehmen untersuchen. Die Honorare für Abschlussprüfungsleistungen stellen ein Surrogat für Prüfungsqualität dar, weil sie Rückschlüsse auf den prüferischen Aufwand zulassen. Darauf basierende Schlussfolgerungen, z.B. in dem Sinne, dass die stark rückläufigen Honorare für Abschlussprüfungsleistungen im DAX30 auf eine geringere Prüfungsqualität hindeuten, müssen aber mit Vorsicht gezogen werden. Prüfungshonorare enthalten zum einem möglicherweise Risikoprämien und die konjunkturelle Entwicklung im Betrachtungszeitraum lässt auf ein im Zeitverlauf fallendes Risiko schliessen. Zum anderen ist es durchaus möglich, dass Prüfungsgesellschaften aus Reputationsgründen am Erhalt von DAX30-Mandaten interessiert sind und deshalb nicht kostendeckende Honorare in Kauf nehmen. Ausserdem sind Quersubventionierungen zwischen Prüfungs- und Nichtprüfungshonoraren denkbar. Schliesslich könnten fallende Honorare für Abschlussprüfungsleistungen auch durch den Einsatz effizienterer Prüfungsmethoden verursacht worden sein. Fallende bzw. konstante Honorare für Abschlussprüfungsleistungen belegen lediglich das die anhaltend hohe Konzentration auf den 
Prüfungsmärkten nicht zu Preissteigerungen geführt hat. Dies schliesst grundsätzlich nicht aus, dass das Ausgangsniveau der Prüfungshonorare im Jahr 2009 nicht marktgerecht, d.h. bereits oligopolistisch überhöht war. Die angestellten Überlegungen zur Unabhängigkeit des Abschlussprüfers basieren ausschliesslich auf der anteiligen Höhe der Honorare für Nichtprüfungsleistungen und damit auf dem Eigeninteresse des Prüfers. In diesem Zusammenhang ist zu berücksichtigen, dass die Unabhängigkeit auch durch die Gefahren einer Selbstprüfung, einer Interessensvertretung oder einer persönlichen Vertrautheit gefährdet werden kann. Das Vorliegen dieser Umstände schlägt sich jedoch nicht in den Abschlussprüferhonoraren nieder. Schliesslich ist erneut auf die im Jahr 2016 in Kraft tretende EU-Verordnung zu Abschlussprüfung von Unternehmen von öffentlichem Interesse zu verweisen. Diese enthält neben der bereits erwähnten Kappung der Beratungshonoraranteile weitere einschneidende Veränderungen, die sich in den Abschlussprüferhonoraren niederschlagen sollten. So beschränkt bspw. Artikel 17 Abs. 1 die Laufzeit des Prüfungsmandats auf 10 Jahre. Mit dieser externen Pflichtrotation dürften erhebliche Änderungen in den Honoraren verknüpft sein. Insofern bietet es sich an, die vorliegende Honorarstudie nach Umsetzung der EU-Verordnung zu wiederholen und somit deren Auswirkung auf die Abschlussprüferhonorare zu beurteilen.

\section{Literaturhinweise}

Antle, R. (1984): Auditor Independence, in: Journal of Accounting Research, Vol. 22, S. 1-20.

Ashbaugh, H./LaFond, R./Mayhwe, B. W. (2003): Do Nonaudit Services Compromise Auditor Independence? Further Evidence, in: The Accounting Review, Vol. 78, No. 3, S. 611-639.

Baldacchino, P. J./Borg, J. (2014): An Analysis of the Development of Audit Fees in Malta, in: The IUP Journal of Accounting Research and Audit Process, Vol. 13, No. 2, S. $27-52$.

Bigus, J./Zimmermann, R.-C. (2008): Non-Audit Fees, Market Leaders and Concentration in the German Audit Market: A Descriptive Analysis, in: International Journal of Auditing, Vol. 12, No. 3, S. 159-179.

Bischof, S. (2006): Anhangangaben zu den Honoraren für Leistungen des Abschlussprüfers, in: Die Wirtschaftsprüfung, Jg. 59, Nr. 11, S. 705-713.

BT-Drucksache 16/10067 (2008): Gesetzentwurf der Bundesregierung - Entwurf eines Gesetzes zur Modernisierung des Bilanzrechts (Bilanzrechtsmodernisierungsgesetz - BilMoG), in: BT-Drucksache 16/10067 vom 30. 07. 2008.

Chan, H. L./DeBoskey, D. G./Hee, K. (2012): Audit Fee Patterns of Big Four and Non-Big Four Firms, in: The CPA Journal, Vol. 82, Issue 10, S. 32-36.

Ciesielski, J. T./Weirich, T. R. (2006): Ups and Downs of Audit Fees Since the Sarbanes-Oxley Act, in: The CPA Journal, Vol. 76, No. 10, S. 28-35.

DCGK (2013): Deutscher Corporate Governance Kodex (in der Fassung vom 13. Mai 2013 mit Beschlüssen aus der Plenarsitzung vom 13. Mai 2013). URL: http://dcgk.de//files/dcgk/ usercontent/de/download/kodex/D_CorGov_Endfassung_2013.pdf (Stand: 1.04.2015).

DeFond, M. L./Raghunandan, K./Subramanyam, K. R. (2002): Do Non-Audit Service Fees Impair Auditor Independence? Evidence from Going Concern Audit Opinions, in: Journal of Accounting Research, Vol. 40, Issue 4, S. 1247-1274.

DeFond, M. L./Zhang, J. (2014): A review of archival auditing research, in: Journal of Accounting and Economics, Vol. 58, No. 2-3, S. 275-326. 
Deutsche Börse (2014a): Historical Index Compositions of the Equity- and Strategy Indices of Deutsche Börse, Version 3.9, April 2014. URL: http://dax-indices.com /DE/MediaLibrary/Document/Historical_Index_Compositions.pdf (Stand: 1.04.2015).

Deutsche Börse (2014b): Leitfaden zu den Aktienindizes der Deutschen Börse, Version 6.28, August 2014. URL: http://dax-indices.com/DE/MediaLibrary/Document /Leitfaden_Aktienindizes.pdf (Stand: 1.04.2015).

Deutsche Börse (2014c): EU-regulierter Markt: Prime Standard - Wesentliche Zulassungsvoraussetzungen und Folgepflichten. URL: http://xetra.com/xetra/dispatch/de/binary/gdb _content_pool/ imported_files/public_files/10_downloads/33_going_being_public/10_products/transparency_factsheets/FS_PS_shares.pdf (Stand: 1.04.2015).

Ebke, W. F. (2013): Kommentierung von $\ 318$ HGB, in: Schmidt, K. (Hrsg.): Münchener Kommentar zum Handelsgesetzbuch, 3. Auflage, München.

Ettredge, M./Fuerherm, E. E./Li, C. (2014): Fee pressure and audit quality, in: Accounting, Organizations and Society, Vol. 39, No. 4, S. 247-263.

Eichenseher, J. W./Danos, P. (1981): The Analysis of Industry-Specific Auditor Concentration: Towards an Explanatory Model, in: Accounting Review, Vol. 56, No. 3, S. 479-492.

Ettredge, M./Greenberg, R. (1990): Determinants of Fee Cutting on Initial Audit Engagements, in: Journal of Accounting Research, Vol. 28, Issue 1, S. 198-210.

EU-Kommission (2002): Empfehlung der Kommission vom 16. Mai 2002: Unabhängigkeit des Abschlussprüfers in der EU - Grundprinzipien (2002/590/EG), in: Amtsblatt der Europäischen Gemeinschaften vom 19.7.2002, L191, S. 22-57. URL: http://eur-lex.europa.eu/legal-content/DE/ AUTO/?uri=uriserv:OJ.L_.2002.191.01.0022. 01.DEU (Stand: 1.04.2015).

EU-Kommission (2010): Europäische Kommission, Grünbuch. Weiteres Vorgehen im Bereich der Abschlussprüfung: Lehren aus der Krise. URL: http://ec.europa.eu/internal_market/consultations/ docs/2010/audit/green_paper_audit_de.pdf (Stand 1.04.2015).

EU-Parlament \& Rat der EU (2004): Vorschlag für eine Richtlinie des Europäischen Parlaments und des Rates über die Prüfung des Jahresabschlusses und des konsolidierten Abschlusses und zur Änderung der Richtlinien 78/660/EWG und 83/349/EWG des Rates. URL: http://eur-lex.europa.eu/legal-content/AUTO/?uri=CELEX:52004PC0177\&qid $=1409$ 771590751\& $\mathrm{rid}=2$ (Stand: 1.04.2015).

EU-Parlament \& Rat der EU (2006): Richtlinie 2006/43/EG des Europäischen Parlaments und des Rates vom 17. Mai 2006 über Abschlussprüfungen von Jahresabschlüssen und konsolidierten Abschlüssen, zur Änderung der Richtlinien 78/660/EWG und 83/349/EWG des Rates und zur Aufhebung der Richtlinie 84/253/EWG des Rates, in: Amtsblatt der Europäischen Gemeinschaften vom 9.6.2006, L157, S. 87-107.

EU-Parlament \& Rat der EU (2014): Verordnung des Europäischen Parlaments und des Rates über spezifische Anforderungen an die Abschlussprüfung bei Unternehmen von öffentlichem Interesse und zur Aufhebung des Beschlusses 2005/909/EG der Kommission, Brüssel.

Frankel, R. M./Johnson, M. F./Nelson K. K. (2002): The Relation between Auditors‘ Fees for Nonaudit Services and Earnings Management, in: The Accounting Review, Vol. 77, Supplement, S. 71-105.

Grottel, B. (2014a): Kommentierung von $\$ 285$ HGB, in: Förschle, G., et al. (Hrsg.): Beck'scher Bilanz-Kommentar, 9. Auflage, München.

Grottel, B. (2014b): Kommentierung von $\$ 314$ HGB, in: Förschle, G., et al. (Hrsg.): Beck'scher Bilanz-Kommentar, 9. Auflage, München. 
Heß, B./Stefani, U. (2012): Ökonomische Konsequenzen der Einschränkung von "Prüfung und Beratung aus einer Hand“, in: Die Wirtschaftsprüfung, Jg. 65, Nr. 22, S. 1177-1186.

IDW RH HFA 1.006 (2005): IDW Rechnungslegungshinweis: Anhangangaben nach $\$ 285$ Satz 1 Nr. 17 HGB bzw. $\$ 314$ Abs. 1 Nr. 9 HGB über das Abschlussprüferhonorar, in: Die Wirtschaftsprüfung, Jg. 58, Nr. 22, S. 1232-1234.

IDW RS HFA 36 (2010): IDW Stellungnahme zur Rechnungslegung: Anhangangaben nach $\mathbb{S} 285$ Nr. 17, 314 Abs. 1 Nr. 9 HGB über das Abschlussprüferhonorar, in: IDW Fachnachrichten 6/2010, S. 245-247.

IDW (2012): WP Handbuch 2012 - Wirtschaftsprüfung, Rechnungslegung, Beratung, Band 1, 14. Aufl., Düsseldorf.

IFAC (2010): Handbook of the Code of Ethics for Professional Accountants, New York.

Kallunki, J.-P./Sahlstörm, P./Zerni, M. (2007): Propensity to Switch Auditors and Strictness of Legal Liability Environment: The Role of Audit Mispricing, in: International Journal of Auditing, Vol. 11, S. 165-185.

Kessler, M. (2013): Kommentierung von $\$ 285$ HGB, in: Hennrichs, J., et al. (Hrsg.): Münchener Kommentar zum Bilanzrecht, München.

Kling, A. (2011): Anhangangaben zur Honorierung des Abschlussprüfers nach dem BilMoG, in: Die Wirtschaftsprüfung, Jg. 64, Nr. 5, S. 209-219.

Köhler, A. G./Marten, K.-U./Ratzinger, N./Wagner, M. (2010): Prüfungshonorare in Deutschland Determinanten und Implikationen, in: Zeitschrift für Betriebswirtschaft, Jg. 80, Issue 1, S. 5-29.

Krauß, P. (2008): Publizität von Abschlussprüferhonoraren bei kapitalmarktorientierten Unternehmen - Zielsetzung und Wirkung der Regelungen im Bilanzrechtsreformgesetz, Sternenfels.

Lenz, H./Bauer, M. (2004): Prüfungs- und Beratungshonorare von Abschlussprüfern deutscher börsennotierter Aktiengesellschaften, in: Die Wirtschaftsprüfung, Jg. 57, Nr. 18, S. 985-998.

Lenz, H./Möller, M./Höhn, B. (2006): Offenlegung der Honorare für Abschlussprüferleistungen im Geschäftsjahr 2005 bei DAX-Unternehmen, in: Betriebs Berater, Jg. 61, Nr. 33, S. 1787-1793.

Lenz, H./Ostrowski, M. (1999): Der Markt für Abschlussprüfungen bei börsennotierten Aktiengesellschaften, in: Die Betriebswirtschaft, Jg. 59, Nr. 3, S. 397-411.

Menon, K./Wiliams, D. D. (2001): Long-Term Trends in Audit Fees, in: Auditing: A Journal of Practice and Theory, Vol. 20, No. 1, S.115-136.

Niehus, R. J. (2002): Corporate Governance: Das Honorar und der Abschlussprüfer - Stärkung der Unabhängigkeit durch Offenlegung?, in: Die Wirtschaftsprüfung, Jg. 55, Nr. 12, S. 616-625.

Petersen, K./Zwirner, C. (2008a): Die Abschlussprüfung im Lichte des BilMoG - Aktualisierung und Internationalisierung, in: Steuern und Bilanzen, S. 50-57.

Petersen, K./Zwirner, C. (2008b): Angabepflicht der Honoraraufwendungen für den Abschlussprüfer - Theoretische und empirische Betrachtungen der Offenlegungserfordernisse zur Stärkung der Prüferunabhängigkeit, in: Die Wirtschaftsprüfung, Jg. 61, Nr. 7, S. 279-291.

Petersen, K./Zwirner, C. (2008c): Abschlussprüfung nach dem Regierungsentwurf zum BilMoG, in: Die Wirtschaftsprüfung, Jg. 61, Nr. 20, S. 967-973.

Petersen, K./Zwirner, C./Boecker, C. (2010): Der Wirtschaftsprüfungsmarkt in Deutschland - Ergebnisse einer Analyse in DAX, MDAX, SDAX und TecDAX, in: KoR - Zeitschrift für kapitalmarktorientierte Rechnungslegung, Jg. 10, Nr. 4, S. 217-224.

Pfitzer, N./Oser, P./Orth, C. (2008): Reform des Aktien-, Bilanz- und Aufsichtsrechts: BilMoG, MoMiG, TUG, EHUG und weitere Reformgesetze, Stuttgart. 
Polt, N./Winter, T. (2004): Der Honoraranspruch des Abschlussprüfers, in: Die Wirtschaftsprüfung, Jg. 57, S. 1127-1135.

Quick, R. (2006): Prüfung, Beratung und Unabhängigkeit des Abschlussprüfers - Eine Analyse der neuen Unabhängigkeitsnormen des HGB im Lichte empirischer Forschungsergebnisse, in: Betriebswirtschaftliche Forschung und Praxis, Jg. 58, Nr. 1, S. 42-61.

Quick, R./Warming-Rasmussen B. (2009): Auditor Independence and the Provision of Non-Audit Services: Perceptions by Germany Investors, in: International Journal of Auditing, Vol. 14, No. 2, S. 141-162.

Quick, R./Wolz, M./Seelbach, M. (1998): Die Struktur des Prüfungsmarktes für deutsche Aktiengesellschaften - Eine empirische Untersuchung der Konzentration auf dem deutschen Wirtschaftsprüfungsmarkt für Aktiengesellschaften, in: Zeitschrift für Betriebswirtschaft, Jg. 68, Nr. 8, S. 779-802.

Reynolds, J. K./Deis Jr., D. R./Francis, J. R. (2004): Professional Service Fees and Auditor Objectivity, in: Auditing: A Journal of Practice and Theory, Vol. 23, No. 1, S. 29-52.

Sattler, M. (2011): Vereinbarkeit von Abschlussprüfung und Beratung - eine empirische Analyse zu den Beratungsleistungen deutscher Abschussprüfer und deren Auswirkungen auf das Ausmaß der Bilanzpolitik ihrer Mandanten, Wiesbaden.

Schmidt, S./Heinz, S. (2014): Kommentierung von $\$ 318$ HGB, in: Förschle, G., et al. (Hrsg.): Beck'scher Bilanz-Kommentar, 9. Auflage, München.

Seetharaman, A./Gul, F. A./Lynn, S. G. (2002): Litigation risk and audit fees: evidence from UK firms cross-listed on US markets, in: Journal of Accounting and Economics, Vol. 33, Issue 1, S. 91-115.

Senger, T./Maier, C. (2013): Kommentierung von $\mathbb{} 314$ HGB, in: Hennrichs, J., et al. (Hrsg.): Münchener Kommentar zum Bilanzrecht, München.

Simon, D. T./Francis, J. R. (1988): The Effects of Auditor Change on Audit Fees: Tests of Price Cutting and Price Recovers, in: The Accounting Review, Vol. 63, No. 2, S. 255-269.

Simunic, D. A. (1980): The pricing of audit services: theory and evidence, in: Journal of Accounting Research, Vol. 18, No. 1, 161-190.

Tate, S. L. (2007): Auditor Change and Auditor Choice in Nonprofit Organizations, in: Auditing: A Journal of Practice and Practice, Vol. 26, Issue 1, S. 47-70.

Umlauf, S. J. (2013): Prüfungs- und Beratungshonorare von Konzernabschlussprüfern - Honorardeterminanten und Unabhängigkeitswahrnehmungen auf dem deutschen Kapitalmarkt, Hamburg.

Velte, P. (2009): Die Vereinbarung des Prüferhonorars durch Aufsichtsrat und Abschlussprüfer als Element der Corporate Governance - Eine normative und empirische Bestandsaufnahme für den deutschen Kapitalmarkt, in: Die Wirtschaftsprüfung, Jg. 62, Nr. 24, S. 1229-1233.

Vogt, E. (2009): Abschlussprüferhaftung gegenüber Dritten in Deutschland und den USA, Baden-Baden.

Wollmert, P./Oser, R./Graupe, F. (2010): Anhangangaben zu den Abschlussprüferhonoraren und zu marktunüblichen Geschäften nach BilMoG, in: Steuern und Bilanzen, S. 123-130.

WPK (2011): Ergebnisse der Honorarumfragen 2010/2011 der WPK. URL: http://www.wpk. de/ mitglieder/praxishinweise/honorarumfrage/ (Stand: 1.04.2015).

Woo, E-S./Koh, H. C. (2001): Factors associated with auditor changes: a Singapore study, in: Accounting and Business Research, Vol. 31, Issue 2, S. 133-144.

Wulf, I. (2010): Aktuelle Neuerungen in der Anhangberichterstattung aus $\mathbb{} 285 \mathrm{HGB}$ nach dem BilMoG, in: Deutsche Steuer-Zeitung, Jg. 98, Nr. 4, S. 405-415. 
Zimmermann, R.-C. (2006): Gestaltungsspielräume bei Veröffentlichung von Abschlussprüferhonoraren im Rahmen des BilReG, in: Zeitschrift für kapitalmarktorientierte Rechnungslegung, S. 273-276.

Zülch, H./Krauß, P./Pronobis, P. (2010): Die Entwicklung von Abschlussprüferhonoraren in Deutschland zwischen 2004 und 2008: Eine empirische Analyse ausgewählter Börsenindizes (DAX, MDAX, SDAX, TecDAX), in: Die Wirtschaftsprüfung, Jg. 63, Nr. 8, S. 397-404.

Heiko Laudemann, B.Sc. Wirtsch.-Ing., ist Masterand am Lehrstuhl für Rechnungswesen, Controlling und Wirtschaftsprüfung an der Technischen Universität Darmstadt

Anschrift: Technische Universität Darmstadt, Fachbereich Rechts- und Wirtschaftswissenschaften, Fachgebiet Rechnungswesen, Controlling und Wirtschaftsprüfung, Hochschulstr. 1, D-64289 Darmstadt, Tel.: +49 (0)6151/16-3423, Fax: +49 (0)6151/16-6034, E-Mail: heiko.laudemann@stud.tu-darmstadt.de

Reiner Quick, Prof. Dr., ist Inhaber des Lehrstuhls für Rechnungswesen, Controlling und Wirtschaftsprüfung an der Technischen Universität Darmstadt und Inhaber einer Teilzeitprofessur für Auditing an der University of Southern Denmark

Anschrift: Technische Universität Darmstadt, Fachbereich Rechts- und Wirtschaftswissenschaften, Fachgebiet Rechnungswesen, Controlling und Wirtschaftsprüfung, Hochschulstr. 1, D-64289 Darmstadt, Tel.: +49 (0)6151/16-3423, Fax: +49 (0)6151/16-6034, E-Mail: quick@bwl.tu-darmstadt.de

Florian Schmidt, M.Sc. Wirtsch.-Inf., ist wissenschaftlicher Mitarbeiter am Lehrstuhl für Rechnungswesen, Controlling und Wirtschaftsprüfung an der Technischen Universität Darmstadt

Anschrift: Technische Universität Darmstadt, Fachbereich Rechts- und Wirtschaftswissenschaften, Fachgebiet Rechnungswesen, Controlling und Wirtschaftsprüfung, Hochschulstr. 1, D-64289 Darmstadt, Tel.: +49 (0)6151/16-7304, Fax: +49 (0)6151/16-6034, E-Mail: fschmidt@bwl.tu-darmstadt.de

Bent Warming-Rasmussen, Prof. Dr., ist Inhaber einer Professur für Wirtschaftsprüfung an der University of Southern Denmark

Anschrift: Department of Entrepreneurship and Relationship Management, University of Southern Denmark, Universtetsparken 1, 6000 Kolding, Denmark, E-Mail: bwr@sam.sdu.dk 